MATHEMATICS OF COMPUTATION

Volume 67, Number 223, July 1998, Pages 965-986

S 0025-5718(98)00954-5

\title{
DISCRETE GAUGE INVARIANT APPROXIMATIONS OF A TIME DEPENDENT GINZBURG-LANDAU MODEL OF SUPERCONDUCTIVITY
}

\author{
QIANG DU
}

\begin{abstract}
We present here a mathematical analysis of a nonstandard difference method for the numerical solution of the time dependent GinzburgLandau models of superconductivity. This type of method has been widely used in numerical simulations of the behavior of superconducting materials. We also illustrate some of their nice properties such as the gauge invariance being retained in discrete approximations and the discrete order parameter having physically consistent pointwise bound.
\end{abstract}

\section{INTRODUCTION}

The phenomenological model of Ginzburg and Landau and its various generalizations [25] have been widely used in numerical studies of the vortex phenomena in both the low $T_{c}$ superconductors as well as the recently discovered high $T_{c}$ superconducting materials. The mathematical analyses of the numerical methods used to solve the Ginzburg-Landau models are mostly confined to the conventional finite difference methods in one space dimension [20] and the finite element methods in two and three dimensions [2], [6], [7], [8], [9], [10]. In practice, a very popular discretization of the Ginzburg-Landau equations is the gauge invariant difference approximation defined on a rectangular grid [3], [4], [14], [15], [16], [17], [18], [19], [21]. Retaining the gauge invariance at the discrete level is analogous with preserving certain conservation laws and physical principles in the discrete approximation. It is often a property favored by physicists who have been using these models in studies of superconductivity. Numerical evidence suggests that the gauge invariant approximation is a valuable approach, but few rigorous mathematical analyses have appeared.

In this paper, we present a mathematical theory for the convergence of a gauge invariant difference approximation of the two dimensional time dependent GinzburgLandau model. The well-posedness of the initial boundary value problems for such a model, the long time asymptotics of solutions and the gauge invariance properties have all been studied in, for example, [5], [11], [13], [24] and the references cited therein. The finite element approximations have also been discussed by various

Received by the editor June 13, 1996 and, in revised form, February 19, 1997.

1991 Mathematics Subject Classification. Primary 65M12, 65M15; Secondary 82D55.

Key words and phrases. Ginzburg-Landau model of superconductivity, time dependent equations, nonstandard difference approximations, gauge invariance, convergence, error estimates.

Research is supported in part by the U. S. National Science Foundation grant MS-9500718 and in part by the HKUST grant DAG 95/96.SC18. 
authors [2], [6], [7], [8]. However, due to the nonlinear coupling, an important pointwise estimate for the discrete finite element approximation is missing from all the analyses. In order to have a meaningful physical interpretation, such an estimate must hold, and indeed, it holds for the exact solution of the time dependent Ginzburg-Landau equations [5]. We show that, for the gauge invariant approximation, the pointwise estimate can be obtained naturally at the discrete level, and rigorous convergence theory as well as error analysis can be established without any unjustified a priori assumption on the numerical solution.

The paper is organized as follows. In section 2, the time dependent GinzburgLandau model is first presented, followed by a description of some useful notation and terminology. Gauge invariant difference approximations for the time dependent Ginzburg-Landau equations are presented in section 3. In section 4, we discuss the properties of the discrete schemes, establish certain a priori estimates and provide an error analysis. Some additional remarks are given in section 5 .

\section{The time Dependent Ginzburg-Landau model and the DISCRETE VECTOR FIELDS}

2.1. The time dependent Ginzburg-Landau model. Let $\kappa$ be the GinzburgLandau parameter, $\eta$ a given positive relaxation parameter, $(0, T)$ the time interval of interest and $\Omega \subset R^{2}$ the region occupied by the superconducting sample. For simplicity, we assume that $\Omega$ is the unit square and the applied magnetic field $H$ is either a constant or a linear function of the spatial variables. The primary variables used in the time dependent Ginzburg-Landau (GL) model are the complex scalarvalued order parameter $\psi$, the real vector-valued magnetic potential $\mathbf{A}$, and the real scalar-valued electric potential $\bar{\Phi}$.

In a non-dimensional form, the time dependent GL model is given by

$$
\frac{\partial \psi}{\partial t}+i \kappa \bar{\Phi} \psi+\left(\frac{i}{\kappa} \nabla+\mathbf{A}\right)^{2} \psi-\psi+|\psi|^{2} \psi=0 \quad \text { in } \Omega \times(0, T),
$$

$$
\eta\left(\frac{\partial \mathbf{A}}{\partial t}+\nabla \bar{\Phi}\right)+\mathbf{c u r l} \operatorname{curl} \mathbf{A}=\frac{i}{2 \kappa}\left(\psi \nabla \psi^{*}-\psi^{*} \nabla \psi\right)-|\psi|^{2} \mathbf{A} \quad \text { in } \Omega \times(0, T),
$$

where $\psi^{*}$ is the complex conjugate of $\psi$. The boundary conditions are

$$
\begin{gathered}
\left(\frac{i}{\kappa} \nabla \psi+\mathbf{A} \psi\right) \cdot \mathbf{n}=0 \quad \text { on } \Gamma \times(0, T), \\
\operatorname{curl} \mathbf{A}=H \quad \text { on } \Gamma \times(0, T), \\
\eta\left(\frac{\partial \mathbf{A}}{\partial t}+\nabla \bar{\Phi}\right) \cdot \mathbf{n}=\mathbf{J} \cdot \mathbf{n} \quad \text { on } \Gamma \times(0, T),
\end{gathered}
$$

where the given applied current $\mathbf{J}$ is constant in space and satisfies $\mathbf{J}=-\operatorname{curl} H$. The initial conditions are:

$$
\psi(\mathbf{x}, 0)=\psi_{0}(\mathbf{x}) \quad \text { and } \quad \mathbf{A}(\mathbf{x}, 0)=\mathbf{A}_{0}(\mathbf{x}) \quad \text { in } \Omega .
$$

We assume that $\psi_{0} \in \mathcal{H}^{1}(\Omega)$ (the space of complex-valued functions whose real and imaginary parts are in the standard Sobolev space $\left.H^{1}(\Omega)\right)$ and $\mathbf{A}_{0} \in \mathbf{H}^{1}(\Omega)$ (the space of $R^{2}$-valued functions whose components are in $H^{1}(\Omega)$ ). In addition, we assume that $\left|\psi_{0}\right| \leq 1, \operatorname{div} \mathbf{A}_{0}=0$ a.e. in $\Omega$ and $\mathbf{A} \cdot \mathbf{n}=0$ on $\Gamma$. 
It is convenient to introduce an auxiliary variable $\Phi_{a}(\mathbf{x}, t)=(\mathbf{J} \cdot \mathbf{x}) / \eta$ and define $\Phi=\bar{\Phi}-\Phi_{a}$ so that the boundary condition (2.1e) may be replaced by the homogeneous boundary condition

$$
\left(\frac{\partial \mathbf{A}}{\partial t}+\nabla \Phi\right) \cdot \mathbf{n}=0 \quad \text { on } \Gamma .
$$

From now on, we use $(\psi, \mathbf{A}, \Phi)$ as the primary variables for the time dependent GL equations and still call $\Phi$ the electric potential. Note that the equations (2.1a-b) can be written as

$$
\begin{aligned}
\frac{\partial \psi}{\partial t}+i \kappa \bar{\Phi} \psi & =-\frac{\partial \mathcal{G}}{\partial \psi}(\psi, \mathbf{A}), \\
\eta\left(\frac{\partial \mathbf{A}}{\partial t}+\nabla \Phi\right) & =-\frac{\partial \mathcal{G}}{\partial \mathbf{A}}(\psi, \mathbf{A}),
\end{aligned}
$$

where $\mathcal{G}$ is the Ginzburg-Landau energy functional ([7], [25]):

$$
\mathcal{G}(\psi, \mathbf{A})=\int_{\Omega}\left(\frac{1}{2}\left|\left(\frac{i}{\kappa} \nabla+\mathbf{A}\right) \psi\right|^{2}+\frac{1}{4}\left(1-|\psi|^{2}\right)^{2}+\frac{1}{2}|\operatorname{curl} \mathbf{A}-H|^{2}\right) d \Omega
$$

The time dependent Ginzburg-Landau equations $(2.1 \mathrm{a}, \mathrm{b})$ with the prescribed boundary conditions are gauge invariant in the sense that if $(\psi, \mathbf{A}, \Phi)$ is a solution to the equations, so is $(\zeta, \mathbf{Q}, \Theta)$ where $\zeta=\psi e^{i \kappa f}, \mathbf{Q}=\mathbf{A}+\nabla f$, and $\Theta=\Phi-\frac{\partial f}{\partial t}$. Questions related to the well-posedness of the above equations and the fixing of gauges have been studied in [4], [11], [13], [24] and the references cited therein. The gauge invariance has been a very desirable property for physicists who have proposed and who have been using these models. It is also favorable to maintain such an invariance property at the discrete level where numerical approximations are made.

2.2. Meshes and discrete vector fields. In order to simplify the presentation and the analysis of the approximation schemes, we now describe a pair of primal and dual meshes (or commonly referred to as a staggered grid). Let the primal mesh $\Sigma$ be a uniform partition of the square $\Omega$ with $N_{0}$ vertices $\left\{\mathbf{x}_{j}\right\}, N_{1}$ edges that are denoted by $\left\{s_{j k}\right\}$ connecting $\mathbf{x}_{j}, \mathbf{x}_{k}$ and $N_{2}$ square cells that are denoted by $\left\{\tau_{j k l m}\right\}$ having four counterclockwise labeled vertices $\mathbf{x}_{j}, \mathbf{x}_{k}, \mathbf{x}_{l}$ and $\mathbf{x}_{m}$. The centers of the cells are denoted by $\left\{\mathbf{x}_{j k l m}\right\}$. Let $h$ be the mesh size. A dual mesh $\Sigma^{\prime}$ is formed by shifting each cell by one half of the mesh spacing in each coordinate direction from the primal mesh (see Figure 1). Centers of the cells in $\Sigma$ become vertices of $\Sigma^{\prime}$ and vice versa. The cell in $\Sigma^{\prime}$ containing $\mathbf{x}_{j}$ is denoted by $\tau_{j}^{\prime}$ and its area is $\left|\tau_{j}^{\prime}\right| . h_{j, k}^{\prime}$ denotes the length of the edge in $\Sigma^{\prime}$ that bisects $s_{j, k} . h_{j, k}^{\prime}=h$ unless both $\mathbf{x}_{j}, \mathbf{x}_{k}$ are on the boundary of $\Omega$. In the latter case, the dual cell $\tau_{j}^{\prime}$ in $\Sigma^{\prime}$ containing the boundary vertex $\mathbf{x}_{j}$ is to be modified to only include the portion of the cell inside $\Omega$ so that $h_{j, k}^{\prime}=h / 2$.

The gauge invariant discretization of the time dependent Ginzburg-Landau equations requires variously defined discrete vector fields. For a vector field $\vec{v} \in U=R^{N_{0}}$ defined by its component $v_{j}$ at each vertex $\mathbf{x}_{j}$, we use the norm

$$
\|\vec{v}\|_{U, p}=\left(\sum_{j}\left|v_{j}\right|^{p}\left|\tau_{j}^{\prime}\right|\right)^{1 / p}, \quad 1 \leq p<\infty \text { and }\|\vec{v}\|_{U, \infty}=\max _{j}\left|v_{j}\right|,
$$



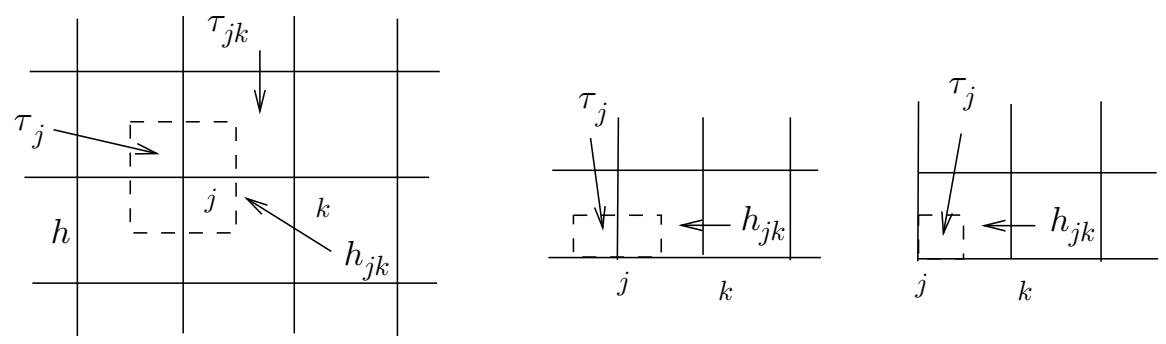

Figure 1. A rectangular grid and its dual. The pictures (from left to right) are for interior vertices, boundary vertices and vertices at corners.

where the sum

$$
\sum_{j}
$$

stands for the sum over all possible vertices $\mathbf{x}_{j}$. We let $\langle\cdot, \cdot\rangle_{U}$ be the inner product corresponding to the norm $\|\cdot\|_{U, 2}$.

For a vector field $\vec{f}$ defined by its value $f_{j k l m}$ at the center of each primal cell $\tau_{j k l m}$ having four vertices $\mathbf{x}_{j}, \mathbf{x}_{k}, \mathbf{x}_{l}, \mathbf{x}_{m}$ which are labeled counterclockwise, we use the convention that $f_{j k l m}=-f_{m l k j}$ if the vertices are labeled clockwise and denote the set of all vectors with this convention by $V$. $V$ is a vector space isomorphic to $R^{N_{2}}$. On $V$, we also define the norms by

$$
\|\vec{f}\|_{V, p}=\left(\sum_{j k l m}\left|f_{j k l m}\right|^{p}\left|\tau_{j k l m}\right|\right)^{1 / p}, \quad 1 \leq p<\infty, \text { and }\|\vec{f}\|_{V, \infty}=\max _{j k l m}\left|f_{j k l m}\right|,
$$

where the sum $\sum_{j k l m}$ stands for the sum over all primal cells $\tau_{j k l m}$ with vertices labeled counterclockwise. The inner product $\langle\cdot, \cdot\rangle_{V}$ corresponds to the norm $\|\cdot\|_{V, 2}$.

For a vector field $\vec{A}$ defined at the midpoint $\mathbf{x}_{j k}=\left(\mathbf{x}_{j}+\mathbf{x}_{k}\right) / 2$ of each edge $s_{j k}$, its component $a_{j k}$ represents a tangential vector $a_{j k} \mathbf{t}_{j k}$. Here, $\mathbf{t}_{j k}=\left(\mathbf{x}_{k}-\mathbf{x}_{j}\right) / h$ is a unit vector in the direction $\mathbf{x}_{j} \mathbf{x}_{k}$. We use the convention that $a_{j k}=-a_{k j}$ and denote the set of all vectors with this convention by $W$. $W$ is a vector space isomorphic to $R^{N_{1}}$. On $W$, the norms are defined by

$$
\|\vec{A}\|_{W, p}=\left(\sum_{j k}\left|a_{j k}\right|^{p} h h_{j k}^{\prime}\right)^{1 / p}, \quad 1 \leq p<\infty, \text { and }\|\vec{A}\|_{W, \infty}=\max _{j k}\left|a_{j k}\right|
$$

where the sum $\sum_{j k}$ stands for the sum over all edges $s_{j k}$. The inner product $\langle\cdot, \cdot\rangle_{W}$ corresponds to the norm $\|\cdot\|_{W, 2}$.

Some discrete operators and discrete inverse inequalities concerning these discrete norms are also useful. For $\vec{u} \in U$, we define $\vec{w}=\nabla \vec{u} \in W$ by

$$
w_{j k}=(\nabla \vec{u})_{j k}=\frac{u_{k}-u_{j}}{h}
$$

on each edge $s_{j k}$.

For $\vec{g} \in V$, we define $\vec{w}=\nabla^{\perp} \vec{g} \in W$ by

$$
w_{j k}=\left(\nabla^{\perp} \vec{g}\right)_{j k}=\frac{g_{j k l m}-g_{j k l^{\prime} m^{\prime}}}{h_{j k}^{\prime}}
$$


on the common edge $s_{j k}$ of two neighboring cells $\tau_{j k l m}$ and $\tau_{j k l^{\prime} m^{\prime}}$ and

$$
w_{j k}=\left(\nabla^{\perp} \vec{v}\right)_{j k}=\frac{1}{h_{j k}^{\prime}} g_{j k l m}
$$

on the edge $s_{j k}$ between two boundary vertices of a boundary cell $\tau_{j k l m}$. Obviously, we have

Lemma 2.1. For $2 \leq p \leq \infty$, there exists some generic positive constant $c$, independent of $h$, such that for any $\vec{u} \in U$,

$$
\|\vec{u}\|_{U, p} \leq c h^{2 / p-1}\|\vec{u}\|_{U, 2} \text {. }
$$

The same inequalities hold when the space $U$ is replaced by $V$ and $W$.

In addition, we also have the following discrete analog of the standard interpolation inequalities in Sobolev spaces.

Lemma 2.2. For $1 \leq p<\infty$, for any $\epsilon>0$, there exists a positive constant $c=c(\epsilon)$ independent of $h$ such that for any $\vec{u} \in U$,

$$
\|\vec{u}\|_{U, p} \leq \epsilon\|\nabla \vec{u}\|_{W, 2}^{2}+c(\epsilon)\|\vec{u}\|_{U, 2}^{2} .
$$

By identifying $\vec{u}$ with a continuous piecewise linear function $u_{h}$ on triangular meshes obtained by dividing each primal cell along a diagonal, the above inequality follows from the continuous version due to the equivalence of norms between $\|\vec{u}\|_{U, p}$ and $\left\|u_{h}\right\|_{L^{p}(\Omega)}$.

\section{Gauge invariant difference approximations}

We present a systematic derivation of the gauge invariant difference approximations for the time dependent Ginzburg-Landau equations in this section.

3.1. The discrete variables. The discrete representations of the primary variables as well as physically interesting variables are defined at the following locations:

$$
\begin{array}{rll}
\text { order parameter } \vec{\psi} & \rightarrow & \text { vertices }\left\{\mathbf{x}_{j}\right\}, \\
\text { electric potential } \vec{\Phi} & \rightarrow & \text { vertices }\left\{\mathbf{x}_{j}\right\}, \\
\text { magnetic potential } \vec{A} & \rightarrow & \text { midpoints of edges }\left\{\mathbf{x}_{j k}\right\}, \\
\text { induced magnetic field } \vec{c} & \rightarrow & \text { center of cells }\left\{\mathbf{x}_{j k l m}\right\}, \\
\text { electric current } \vec{J} & \rightarrow & \text { midpoints of edges }\left\{\mathbf{x}_{j k}\right\} .
\end{array}
$$

Let $\vec{A} \in W$ be a vector field defined at the midpoint of each edge. The circulation in a typical cell $\tau_{j k l m}$ with four vertices $\mathbf{x}_{j}, \mathbf{x}_{k}, \mathbf{x}_{l}, \mathbf{x}_{m}$ is given by

$$
c_{j k l m}:=\left(a_{j k}+a_{k l}+a_{l m}+a_{m j}\right) / h .
$$

The above can be rewritten in matrix terms:

$$
C \vec{A}=\vec{c},
$$

where $C$ may be viewed as an approximation of the operator curl. We approximate the divergence on each dual cell $\tau_{j}^{\prime}$ containing the vertex $\mathbf{x}_{j}$ by

$$
d_{j}:=\frac{1}{\left|\tau_{j}^{\prime}\right|} \sum_{k \rightarrow j} a_{j k} h_{j k}^{\prime}
$$


where the sum $\sum_{k \rightarrow j}$ stands for the sum over all neighboring vertices $\mathbf{x}_{k}$ of $\mathbf{x}_{j}$. We again have the matrix form

$$
D \vec{A}=\vec{d}
$$

where $D$ may be viewed as an approximation of the operator div.

A few technical results concerning the discrete approximations of div and curl are needed for later discussions. First, direct calculation gives a discrete analog of the integration by part formula and the orthogonality of vector fields:

Lemma 3.1. Let $\vec{u}, \vec{v} \in W, D, C$ be matrices defined as in (3.3),(3.5), and $\nabla, \nabla^{\perp}$ defined as in (2.6), (2.7a-b). Then

$$
\begin{array}{cl}
\langle D \vec{u}, \vec{f}\rangle_{U} & =-\langle\vec{u}, \nabla \vec{f}\rangle_{W} \quad \forall \vec{f} \in U, \\
\langle C \vec{u}, \vec{g}\rangle_{V}=\left\langle\vec{u}, \nabla^{\perp} \vec{g}\right\rangle_{W} & \forall \vec{g} \in V, \\
C \nabla \vec{f}=\overrightarrow{0} \quad \forall \vec{f} \in U \quad \text { and } \quad D \nabla^{\perp} \vec{g}=\overrightarrow{0} \quad \forall \vec{g} \in V .
\end{array}
$$

Next, the following inequality can be verified using similar arguments given in [22], [23] (the discrete operators are the dual versions of those defined in [22], [23]; see also [12] for similar results for unstructured triangular grids):

Lemma 3.2. Let $2 \leq p<\infty$. For small $h$, there exists a positive constant $c$ independent of $h$ such that

$$
\|\vec{u}\|_{W, p} \leq c\left(\|D \vec{u}\|_{U, 2}+\|C \vec{u}\|_{V, 2}\right), \quad \forall \vec{u} \in W .
$$

3.2. The approximation of the energy functional. The crucial point in maintaining the gauge invariance in the approximation is to approximate the integral of $\left|\frac{i}{\kappa} \nabla \psi+\mathbf{A} \psi\right|^{2}$ on a primal cell $\tau_{1234}$ by

$$
\frac{h^{2}}{2}\left(\left|\alpha_{12}\right|^{2}+\left|\alpha_{23}\right|^{2}+\left|\alpha_{34}\right|^{2}+\left|\alpha_{41}\right|^{2}\right)
$$

where

$$
\alpha_{j k}=\frac{i}{\kappa} \frac{\psi_{k} \exp \left(-i \kappa a_{j k} h\right)-\psi_{j}}{h}
$$

and $\exp \left(-i \kappa a_{j k} h\right)$ is often called a link variable [1], [14]. Coupling (3.2), (3.11) with the use of a one-point integration rule for the integral of $\left(1-|\psi|^{2}\right)^{2} / 4$ over each dual cell $\tau_{j}^{\prime}$, we arrive at the following discrete formulation of the GL functional:

$$
\begin{aligned}
& \mathcal{G}^{h}(\vec{\psi}, \vec{A})=\frac{1}{2} \sum_{j k} \frac{h h_{j k}^{\prime}}{\kappa^{2}}\left|\frac{\psi_{k} \exp \left(-i \kappa a_{j k} h\right)-\psi_{j}}{h}\right|^{2} \\
& \quad+\sum_{j} \frac{\left|\tau_{j}^{\prime}\right|}{4}\left(1-\left|\psi_{j}\right|^{2}\right)^{2}+\frac{1}{2} \sum_{j k l m}\left(a_{j k}+a_{k l}+a_{l m}+a_{m j}-H_{j k l m} h\right)^{2}
\end{aligned}
$$

where $H_{j k l m}$ is the component of $\vec{H} \in V$ which is equal to the value of $H$ at the center of the cell $\tau_{j k l m}$ with vertices $\mathbf{x}_{j}, \mathbf{x}_{k}, \mathbf{x}_{l}, \mathbf{x}_{m}$ labeled counterclockwise. 
3.3. The semi-discrete gauge invariant approximation. For all vertices $\left\{\mathbf{x}_{j}\right\}$ in $\Sigma$, let $\bar{\Phi}_{j}=\Phi_{j}+\Phi_{a}\left(\mathbf{x}_{j}, t\right)$. The semi-discrete gauge invariant difference approximation is defined by

$$
\frac{\partial \psi_{j}}{\partial t}+i \kappa \bar{\Phi}_{j} \psi_{j}=-\frac{1}{\left|\tau_{j}^{\prime}\right|} \frac{\partial \mathcal{G}^{h}}{\partial \psi_{j}}(\vec{\psi}, \vec{A})
$$

at all the vertices $\left\{\mathbf{x}_{j}\right\}$ in $\Sigma$, and

$$
\eta\left(\frac{\partial a_{j k}}{\partial t}+\frac{\Phi_{k}-\Phi_{j}}{h}\right)=-\frac{1}{h h_{j k}^{\prime}} \frac{\partial \mathcal{G}^{h}}{\partial a_{j k}}(\vec{\psi}, \vec{A})
$$

at the midpoints $\left\{\mathbf{x}_{j k}\right\}$ of all edges in $\Sigma$. The initial conditions are defined as

$$
\psi_{j}(0)=\psi_{0}\left(\mathbf{x}_{j}\right) \quad \text { or } \quad \psi_{j}(0)=\frac{1}{\left|\tau_{j}^{\prime}\right|} \int_{\tau_{j}} \psi_{0}(\mathbf{x}) d \mathbf{x}
$$

at all vertices and the components of $\vec{A}(0)$ form the solution of

$$
C \vec{A}(0)=\vec{c}(0) \in V
$$

and

$$
D \vec{A}(0)=\overrightarrow{0} \in U
$$

where the components of $\vec{c}(0)$ are defined by

$$
c_{j k l m}(0)=\frac{1}{\left|\tau_{j k l m}\right|} \int_{\tau_{j k l m}} \operatorname{curl} \mathbf{A}_{0} d \tau,
$$

in all the cells and $C$ and $D$ are matrices defined as in (3.3) and (3.5).

Given a discrete vector field $\vec{f}(t) \in U$ for $t$ in a given time interval $[0, T]$, the above scheme is invariant under any discrete gauge transformation $\mathcal{T}_{f}$ defined by $\mathcal{T}_{f}(\vec{\psi}, \vec{A}, \vec{\Phi})=(\vec{\zeta}, \vec{Q}, \vec{\Theta})$ where the components of $(\vec{\zeta}, \vec{Q}, \vec{\Theta})$ are given by

$$
\zeta_{j}=\psi_{j} e^{i \kappa f_{j}}, \quad q_{j k}=a_{j k}+\frac{f_{k}-f_{j}}{h} \quad \text { and } \quad \Theta_{j}=\Phi_{j}-\frac{\partial f_{j}}{\partial t},
$$

at the vertices or edges.

3.4. The fully-discrete gauge invariant approximation. There are various discrete-in-time schemes, for example, the explicit forward Euler methods have been used in many simulations. Here, we present a modified backward Euler method. Such a method has unconditional stability.

For simplicity, we take a uniform time step size $\Delta t$ on a given time interval $[0, T]$, although the theory remains valid even if variable step size is allowed. Let $\bar{\Phi}_{j}^{n}=\Phi_{j}^{n}+\Phi_{a j}^{n}$ where $\Phi_{a j}^{n}=\Phi_{a}\left(\mathbf{x}_{j}, n \Delta t\right)$ for all vertices $\mathbf{x}_{j}$ at all time steps. The gauge invariant backward Euler scheme is given by

$$
\begin{aligned}
\frac{\left.\psi_{j}^{n}-\psi_{j}^{n-1} \exp \left(-i \kappa \bar{\Phi}_{j}^{n} \Delta t\right)\right)}{\Delta t} & =-\frac{1}{\left|\tau_{j}^{\prime}\right|} \frac{\partial \mathcal{G}^{h}}{\partial \psi_{j}^{n}}\left(\vec{\psi}^{n}, \vec{A}^{n}\right), \\
\eta\left(\frac{a_{j k}^{n}-a_{j k}^{n-1}}{\Delta t}+\frac{\Phi_{k}^{n}-\Phi_{j}^{n}}{h}\right) & =-\frac{1}{h h_{j k}^{\prime}} \frac{\partial \mathcal{G}^{h}}{\partial a_{j k}^{n}}\left(\vec{\psi}^{n}, \vec{A}^{n}\right)
\end{aligned}
$$


for $n=1,2, \ldots, N=T / \Delta t$. The initial conditions are given by

$$
\psi_{j}^{0}=\psi_{0}\left(\mathbf{x}_{j}\right) \quad \text { or } \quad \psi_{j}^{0}=\frac{1}{\left|\tau_{j}^{\prime}\right|} \int_{\tau_{j}} \psi_{0}(\mathbf{x}) d \mathbf{x}
$$

and the components $a_{j k}^{0}$ of $\vec{A}^{0}$ form the solution of

$$
\begin{gathered}
C \vec{A}^{0}=\vec{c}(0) \in V, \\
D \vec{A}^{0}=\overrightarrow{0} \in U
\end{gathered}
$$

where the components of $\vec{c}(0)$ are defined by (3.13f) and $C, D$ are defined as in (3.3) and (3.5). Other interpolation techniques for the order parameter may also be used if we only have $\psi_{0} \in \mathcal{H}^{1}(\Omega)$.

First of all, let us state the discrete gauge invariance property of the above scheme. Given $\vec{f} \in\left[R^{N_{0}}\right]^{N}$, with $f_{j}^{0}=0$ for any $j$. Let $\mathcal{T}_{f}^{h}(\vec{\psi}, \vec{A}, \vec{\Phi})=(\vec{\zeta}, \vec{Q}, \vec{\Theta})$ where the components of $(\vec{\zeta}, \vec{Q}, \vec{\Theta})$ satisfy

$$
\zeta_{j}^{n}=\psi_{j}^{n} e^{i \kappa f_{j}^{n}}, \quad \Theta_{j}^{n}=\Phi_{j}^{n}-\frac{f_{j}^{n}-f_{j}^{n-1}}{\Delta t} \quad \text { and } \quad q_{j k}^{n}=a_{j k}^{n}+\frac{f_{k}^{n}-f_{j}^{n}}{h}
$$

at vertices or edges. Then, we have

Proposition 3.4. For $\vec{f}^{n} \in R^{N_{0}}$ for $0 \leq n \leq N$ with $f_{j}^{0}=0$ for any $j$. If $(\vec{\psi}, \vec{A}, \vec{\Phi})$ is a solution of (3.14a-b) with initial conditions (3.14c-e), then so is $\mathcal{T}_{f}^{h}(\vec{\psi}, \vec{A}, \vec{\Phi})$.

When performing numerical simulations, the gauge of the solution should be fixed. The above properties, however, make it flexible in selecting a suitable gauge for the convenience of a particular simulation. There are various choices to fix the gauge (see [5] for the continuous analog). A few possibilities are discussed below along with results on the existence of discrete solutions.

3.5. The London gauge. The London gauge is obtained by letting the vector magnetic potential be divergence free, i.e.,

$$
\sum_{k \rightarrow j} a_{j k}^{n} h_{j k}^{\prime}=0
$$

or $D \vec{A}^{n}=\overrightarrow{0}$. In the London gauge, three variables $(\vec{\psi}, \vec{A}, \vec{\Phi})$ need to be solved and the computational cost is often increased. There is no existing numerical simulation of the time dependent models using the London gauge (even though the corresponding Coulomb gauge is widely used in steady state simulations). We thus ignore further discussions of the London gauge.

3.6. The zero electric potential gauge. For the zero electric potential gauge, we require $\vec{\Phi}^{n}=\overrightarrow{0}$, i.e., $\Phi_{j}^{n}=0$ for all $n, j$. This can be achieved by letting

$$
\frac{f_{j}^{n}-f_{j}^{n-1}}{\Delta t}=\Phi_{j}^{n}
$$

with $\vec{f}^{0}=\overrightarrow{0}$. Note that this implies $\bar{\Phi}_{j}^{n}=\Phi_{a}\left(\mathbf{x}_{j}\right)$.

In actual implementation, the solution in the zero electric potential gauge at the $n$-th time step may be obtained by solving for the global minimizer of the following variational problem:

$$
\min _{(\vec{\psi}, \vec{A}) \in U \times W} \mathcal{G}^{h, n}(\vec{\psi}, \vec{A})
$$


where

$$
\mathcal{G}^{h, n}(\vec{\psi}, \vec{A})=\mathcal{G}^{h}(\vec{\psi}, \vec{A})+\frac{1}{\Delta t}\|\vec{\psi}-\vec{\zeta}\|_{U, 2}^{2}+\frac{\eta}{\Delta t}\left\|\vec{A}-\vec{A}^{n-1}\right\|_{W, 2}^{2}
$$

for any $(\vec{\psi}, \vec{A}) \in U \times W$ and $\vec{\zeta}$ be defined by

$$
\zeta_{j}=\psi_{j}^{n-1} \exp \left(-i \kappa \Phi_{a j}^{n} \Delta t\right) .
$$

It is obvious that $\mathcal{G}^{h . n}$ is continuous and bounded below by

$$
\frac{1}{\Delta t}\|\vec{\psi}-\vec{\zeta}\|_{U, 2}^{2}+\frac{\eta}{\Delta t}\left\|\vec{A}-\vec{A}^{n-1}\right\|_{W, 2}^{2},
$$

so the minimum is achieved in a bounded set for given $h$ and $\Delta t$. Consequently, we have

Lemma 3.5. Given $h, \Delta t$ and $\left(\vec{\psi}^{n-1}, \vec{A}^{n-1}\right)$, there exists at least one solution to the gauge invariant difference approximation in the zero electric potential gauge.

Naturally, the above result implies the existence of solutions to the fully-discrete scheme under any gauge choice by applying proper gauge transformations.

3.7. The Lorentz coupled potential gauge. The Lorentz coupled potential gauge is the gauge for which the discrete vector potential and the discrete electric potential are to be related by $\vec{\Phi}^{n}=\lambda D \vec{A}^{n}$, or

$$
\Phi_{j}^{n}=\frac{\lambda}{\left|\tau_{j}^{\prime}\right|}\left(\sum_{k \rightarrow j} a_{j k}^{n} h_{j k}^{\prime}\right)
$$

where $\lambda \geq 0$ is a given constant. This includes both $\S 3.5(\lambda=\infty)$ and $\S 3.6(\lambda=0)$.

Using similar ideas as in [5] for the continuous case (see also [13] for related discussion), the construction of the gauge transformation $\mathcal{T}_{f}^{h}$ is given by

$$
\frac{f_{j}^{n}-f_{j}^{n-1}}{\Delta t}-\frac{\lambda}{\left|\tau_{j}^{\prime}\right|} \sum_{k \rightarrow j} \frac{f_{k}^{n}-f_{j}^{n}}{h_{k j}} h_{k j}^{\prime}=\Phi_{j}^{n}+\lambda \frac{1}{\left|\tau_{j}^{\prime}\right|} \sum_{k \rightarrow j} a_{j k}^{n} h_{j k}^{\prime}
$$

and $\vec{f}^{0}=\overrightarrow{0}$. The case $\lambda=1$ is often referred to as the Lorentz gauge. We take this gauge as an example for our theoretical studies given in later sections.

Using gauge transformations, any solution of the gauge invariant approximation (3.14) can be transformed into a solution in the Lorentz gauge, so it follows from Lemma 3.5 that

Lemma 3.6. Given $h, \Delta t$ and $\left(\vec{\psi}^{n-1}, \vec{A}^{n-1}\right)$, there exists at least one solution to the gauge invariant difference approximation in the Lorentz gauge.

\section{Analysis of Gauge inVARIAnt DifFEREnCE APPROXIMATIONS}

We analyze the convergence of the fully-discrete approximation in the Lorentz gauge. The key steps include the derivation of an a priori pointwise estimate and an energy estimate. For an interesting comparison with their continuous counterparts as well as their finite element versions, one may consult, for example, [5], [6]. Here, we focus on the modifications necessitated by the discrete approximations. The stability estimates are obtained by comparing solutions with perturbations which in turn imply the convergence and error estimates. 
4.1. A pointwise bound on the fully-discrete solution. The time dependent GL equations presented in (2.1a-f) are non-dimensionalized; it can be shown [5] that the magnitude of the order parameter, $|\psi|$, remains between 0 and 1 with $\psi=0$ representing the normal state and $|\psi|=1$ representing an ideal superconducting state. Here, we prove the discrete maximum principle which is presented as a property of the gauge invariant scheme (3.14a-e), that is, it holds for solutions in any given gauge.

Lemma 4.1. Let $\left(\vec{\psi}^{n}, \vec{A}^{n}\right)$ satisfy (3.14a-e). If $\left|\psi^{0}(\mathbf{x})\right| \leq 1$ for almost all $\mathbf{x} \in \Omega$, we have $\left\|\vec{\psi}^{n}\right\|_{U, \infty} \leq 1$ for all $n>0$.

Proof. By the construction of the initial approximation, we have $\left\|\vec{\psi}^{0}\right\|_{U, \infty} \leq 1$. Assume that $\left|\psi_{j}^{n}\right|=\left\|\overrightarrow{\psi^{n}}\right\|_{V, \infty}$ for some $n, j$ and $\left|\psi_{j}^{n}\right|>1$ while $\left\|\vec{\psi}^{k}\right\|_{V, \infty} \leq 1$ for all $k<n$. Notice that (3.14a) has the following form

$$
\begin{gathered}
\frac{\psi_{j}^{n}-\psi_{j}^{n-1} \exp \left(-i \kappa \bar{\Phi}_{j}^{n} \Delta t\right)}{\Delta t}=\left(1-\left|\psi_{j}^{n}\right|^{2}\right) \psi_{j}^{n} \\
+\frac{1}{\left|\tau_{j}^{\prime}\right|} \sum_{k \rightarrow j} \frac{h_{j k}^{\prime}}{\kappa^{2}}\left(\frac{\psi_{k}^{n} \exp \left(-i \kappa a_{j k}^{n} h\right)-\psi_{j}^{n}}{h}\right) .
\end{gathered}
$$

Multiplying the above equation by $\Delta t \psi_{j}^{n *}$ and rearranging terms, we get

$$
\begin{gathered}
\left(1+\Delta t\left(\left|\psi_{j}^{n}\right|^{2}-1\right)+\frac{\Delta t}{\left|\tau_{j}^{\prime}\right|} \sum_{k \rightarrow j} \frac{h_{j k}^{\prime}}{h \kappa^{2}}\right)\left|\psi_{j}^{n}\right|^{2}=\psi_{j}^{n-1} \psi_{j}^{n *} \exp \left(-i \kappa \bar{\Phi}_{j}^{n} \Delta t\right) \\
+\frac{\Delta t}{\left|\tau_{j}^{\prime}\right|} \sum_{k \rightarrow j} \frac{h_{j k}^{\prime}}{\kappa^{2}}\left(\frac{\psi_{k}^{n} \psi_{j}^{n *} \exp \left(-i \kappa a_{j k}^{n} h\right)}{h}\right) .
\end{gathered}
$$

Note that for any $j$,

$$
\frac{1}{\left|\tau_{j}^{\prime}\right|} \sum_{k \rightarrow j} \frac{h_{j k}^{\prime}}{h}=\frac{4}{h^{2}} .
$$

By the assumption on $\left|\psi_{j}^{n}\right|$, we get

$$
\begin{aligned}
\left(1+\frac{4 \Delta t}{h^{2} \kappa^{2}}\right)\left|\psi_{j}^{n}\right|^{2} & <\left|\psi_{j}^{n-1}\right|\left|\psi_{j}^{n}\right|+\frac{\Delta t}{\left|\tau_{j}^{\prime}\right|} \sum_{k \rightarrow j} \frac{h_{j k}^{\prime}}{h \kappa^{2}}\left|\psi_{k}^{n}\right|\left|\psi_{j}^{n}\right| \\
& \leq\left|\psi_{j}^{n-1}\right|\left|\psi_{j}^{n}\right|+\frac{4 \Delta t}{\kappa^{2} h^{2}}\left|\psi_{j}^{n}\right|^{2} .
\end{aligned}
$$

Thus, $\left|\psi_{j}^{n}\right|<\left|\psi_{j}^{n-1}\right| \leq 1$. This contradiction proves the lemma.

Remark. The above result insures that the magnitude square of the discrete order parameter has proper physical interpretation as the density of superconducting carriers. The same estimate holds for solutions of (3.14a-e) in any given gauge as well as the semi-discrete approximation presented in (3.13a-b).

4.2. A priori estimates for approximation in the Lorentz gauge. Given the solution $\left(\vec{\psi}^{n-1}, \vec{A}^{n-1}\right)$ of the $n-1$ time step and setting $\vec{\Phi}^{n}=-D \overrightarrow{A^{n}}$ in (3.14a)(3.14b), the difference approximation in the Lorentz gauge at the $n$-th time step is 
given by

$$
\begin{gathered}
\frac{\psi_{j}^{n}-\psi_{j}^{n-1} \exp \left(-i \kappa\left(\Phi_{a j}^{n}+d_{j}^{n}\right) \Delta t\right)}{\Delta t}=\left(1-\left|\psi_{j}^{n}\right|^{2}\right) \psi_{j}^{n} \\
+\frac{1}{\left|\tau_{j}^{\prime}\right|} \sum_{k \rightarrow j} \frac{h_{j k}^{\prime}}{\kappa^{2}}\left(\frac{\psi_{k}^{n} \exp \left(-i \kappa a_{j k}^{n} h\right)-\psi_{j}^{n}}{h}\right), \\
\eta\left(\frac{a_{j k}^{n}-a_{j k}^{n-1}}{\Delta t}\right)= \\
+\left(\nabla \vec{d}^{n}\right)_{j k}-\left(\nabla^{\perp}\left(\vec{c}^{n}-\vec{H}\right)\right)_{j k} \\
+\frac{1}{h \kappa} \Re\left\{i \psi_{k}^{n *} \psi_{j}^{n} \exp \left(i \kappa a_{j k}^{n} h\right)\right\},
\end{gathered}
$$

where

$$
\vec{d}^{n}=D \vec{A}^{n}, \quad \text { and } \quad \vec{c}^{n}=C \vec{A}^{n}
$$

and $\nabla, \nabla^{\perp}$ are defined as in (2.6) and (2.7a-b). The initial conditions are defined in $(3.14 \mathrm{c}-\mathrm{e})$ as before.

The existence of solutions of $(4.4 \mathrm{a}-\mathrm{b})$ follows from Lemma 3.6. We show later that the solution is unique if we take small step size $\Delta t$. Similar to [7], [12], in order to study the properties of the above scheme, we define the following modified free energy functional:

$$
\mathcal{F}^{h}\left(\vec{\psi}^{h}, \vec{A}^{h}\right)=\mathcal{G}^{h}\left(\vec{\psi}^{h}, \vec{A}^{h}\right)+\sum_{j} \frac{1}{2\left|\tau_{j}^{\prime}\right|}\left(\sum_{k \rightarrow j} a_{j k} h_{j k}^{\prime}\right)^{2} .
$$

Then, one may rewrite (4.4a-b) as

$$
\frac{\left.\psi_{j}^{n}-\psi_{j}^{n-1} \exp \left(-i \kappa\left(\bar{\Phi}_{j}^{n}+d_{j}^{n}\right) \Delta t\right)\right)}{\Delta t}=-\frac{1}{\left|\tau_{j}^{\prime}\right|} \frac{\partial \mathcal{F}^{h}}{\partial \psi_{j}^{n}}\left(\vec{\psi}^{n}, \vec{A}^{n}\right),
$$

and

$$
\eta \frac{a_{j k}^{n}-a_{j k}^{n-1}}{\Delta t}=-\frac{1}{h h_{j k}^{\prime}} \frac{\partial \mathcal{F}^{h}}{\partial a_{j k}^{n}}\left(\vec{\psi}^{n}, \vec{A}^{n}\right) .
$$

To establish the energy bounds, we have

Lemma 4.2. There exists a positive constant $c$, which depends on the given parameters $\kappa, \eta$ and the final time $T$, but is independent of $h$ and $\Delta t$, such that for small $\Delta t$,

$$
\begin{gathered}
\mathcal{F}^{h}\left(\vec{\psi}^{n}, \vec{A}^{n}\right)+\sum_{m=1}^{n} \frac{1}{\Delta t}\left\{\left\|\vec{\psi}^{m}-\vec{\psi}^{m-1}\right\|_{U, 2}^{2}+\left\|\vec{A}^{m}-\vec{A}^{m-1}\right\|_{W, 2}^{2}\right\} \\
\leq c \mathcal{F}^{h}\left(\vec{\psi}^{0}, \vec{A}^{0}\right)+c|\mathbf{J}|^{2} .
\end{gathered}
$$

Proof. We consider

$$
\begin{aligned}
\mathcal{F}^{h}\left(\vec{\psi}^{n}, \vec{A}^{n}\right)-\mathcal{F}^{h}\left(\vec{\psi}^{n-1}, \vec{A}^{n-1}\right) \\
\quad-\Re\left\{\frac{\partial \mathcal{F}^{h}}{\partial \vec{\psi}^{n}}\left(\vec{\psi}^{n}, \vec{A}^{n}\right)\left(\vec{\psi}^{n}-\vec{\psi}^{n-1}\right)+\frac{\partial \mathcal{F}^{h}}{\partial \vec{A}^{n}}\left(\vec{\psi}^{n}, \vec{A}^{n}\right)\left(\vec{A}^{n}-\vec{A}^{n-1}\right)\right\}
\end{aligned}
$$


term by term. First of all, by Lemma 3.1, we have

$$
\begin{aligned}
\frac{1}{2}\left(\left\|D \vec{A}^{n}\right\|_{U, 2}^{2}+\left\|C \vec{A}^{n}-\vec{H}\right\|_{V, 2}^{2}-\left\|D \vec{A}^{n-1}\right\|_{U, 2}^{2}-\left\|C \vec{A}^{n-1}-\vec{H}\right\|_{V, 2}^{2}\right) & \\
& +\left\langle\nabla \vec{d}^{n}, \vec{A}^{n}-A^{\overrightarrow{n-1}}\right\rangle_{W}-\left\langle\nabla^{\perp}\left(\vec{c}^{n}-\vec{H}\right), \vec{A}^{n}-A^{\overrightarrow{n-1}}\right\rangle_{W} \\
=- & \frac{1}{2}\left(\left\|D\left(\vec{A}^{n}-\vec{A}^{n-1}\right)\right\|_{U, 2}^{2}+\left\|C\left(\vec{A}^{n}-\vec{A}^{n-1}\right)\right\|_{V, 2}^{2}\right) .
\end{aligned}
$$

Next, since $\left(1+z^{2}\right)^{2}$ is a convex function of $z$, simple calculation gives

$$
\begin{aligned}
& \frac{\left|\tau_{j}^{\prime}\right|}{4}\left[\left(1-\left|\psi_{j}^{n}\right|^{2}\right)^{2}-\left(1-\left|\psi_{j}^{n-1}\right|^{2}\right)^{2}-\Re\left\{\left(1-\left|\psi_{j}^{n}\right|^{2}\right) \psi_{j}^{n}\left(\psi_{j}^{n *}-\psi_{j}^{n-1 *}\right)\right\}\right] \\
& \quad \leq\left|\tau_{j}^{\prime}\right|\left|\psi_{j}^{n}-\psi_{j}^{n-1}\right|^{2}
\end{aligned}
$$

The term that needs some calculation is

$$
\begin{aligned}
\mathrm{I}_{1}:=\frac{1}{2} \sum_{j k} & \frac{h h_{j k}^{\prime}}{\kappa^{2}}\left[\frac{\left|\psi_{k}^{n} \exp \left(-i \kappa a_{j k}^{n} h\right)-\psi_{j}^{n}\right|^{2}}{h^{2}}-\frac{\left|\psi_{k}^{n-1} \exp \left(-i \kappa a_{j k}^{n-1} h\right)-\psi_{j}^{n-1}\right|^{2}}{h^{2}}\right] \\
& +\sum_{j} \sum_{k \rightarrow j} \frac{h_{j k}^{\prime}}{\kappa^{2}} \Re\left\{\left(\frac{\psi_{k}^{n} \exp \left(-i \kappa a_{j k}^{n} h\right)-\psi_{j}^{n}}{h}\right)\left(\psi_{j}^{n *}-\psi_{j}^{n-1 *}\right)\right\} \\
& +\sum_{j k} \frac{h_{j k}^{\prime}}{\kappa} \Re\left\{i \psi_{k}^{n *} \psi_{j}^{n} \exp \left(i \kappa a_{j k}^{n} h\right)\left(a_{j k}^{n}-a_{j k}^{n-1}\right)\right\} .
\end{aligned}
$$

By expanding terms, we get

$$
\begin{aligned}
\mathrm{I}_{1}= & \frac{1}{2} \sum_{j k} \frac{h_{j k}^{\prime}}{h \kappa^{2}}\left[\left|\psi_{k}^{n}\right|^{2}+\left|\psi_{j}^{n}\right|^{2}-2 \Re\left\{\psi_{k}^{n} \exp \left(-i \kappa a_{j k}^{n} h\right) \psi_{j}^{n *}\right\}-\left|\psi_{k}^{n-1}\right|^{2}-\left|\psi_{j}^{n-1}\right|^{2}\right. \\
& +2 \Re\left\{\psi_{k}^{n-1} \exp \left(-i \kappa a_{j k}^{n-1} h\right) \psi_{j}^{n-1 *}\right\}+2 \Re\left\{\psi_{k}^{n} \exp \left(-i \kappa a_{j k}^{n} h\right) \psi_{j}^{n *}\right\}-2\left|\psi_{j}^{n}\right|^{2} \\
& -2 \Re\left\{\psi_{k}^{n} \exp \left(-i \kappa a_{j k}^{n} h\right) \psi_{j}^{n-1 *}\right\}+2 \Re\left\{\psi_{j}^{n} \psi_{j}^{n-1 *}\right\}+2 \Re\left\{\psi_{j}^{n} \exp \left(i \kappa a_{j k}^{n} h\right) \psi_{k}^{n *}\right\} \\
& -2\left|\psi_{k}^{n}\right|^{2} 2 \Re\left\{\psi_{j}^{n} \exp \left(i \kappa a_{j k}^{n} h\right) \psi_{k}^{n-1 *}\right\}+2 \Re\left\{\psi_{k}^{n} \psi_{k}^{n-1 *}\right\} \\
& \left.+2 \Re\left\{i \psi_{k}^{n *} \psi_{j}^{n} \exp \left(i \kappa a_{j k}^{n} h\right) \kappa\left(a_{j k}^{n}-a_{j k}^{n-1}\right) h\right\}\right] .
\end{aligned}
$$

Combining like terms, we have

$$
\begin{aligned}
\mathrm{I}_{1}=\frac{1}{2} \sum_{j k} & \frac{h_{j k}^{\prime}}{h \kappa^{2}}\left[2 \Re\left\{\psi_{k}^{n-1} \psi_{j}^{n-1 *}\left(\exp \left(-i \kappa a_{j k}^{n-1} h\right)-\exp \left(i \kappa a_{j k}^{n} h\right)\right)\right\}\right. \\
+ & 2 \Re\left\{i \psi_{k}^{n *} \psi_{j}^{n} \exp \left(i \kappa a_{j k}^{n} h\right) \kappa\left(a_{j k}^{n}-a_{j k}^{n-1}\right) h\right\} \\
& \left.-\left|\left(\psi_{k}^{n}-\psi_{k}^{n-1}\right) \exp \left(-i \kappa a_{j k}^{n} h\right)-\left(\psi_{j}^{n}-\psi_{j}^{n-1}\right)\right|^{2}\right] .
\end{aligned}
$$


Rearranging the terms, we get

$$
\begin{aligned}
\mathrm{I}_{1}= & \sum_{j k} \frac{h h_{j k}^{\prime}}{\kappa^{2}}\left[\Re \left\{\left(\frac{\psi_{k}^{n-1} \exp \left(-i \kappa a_{j k}^{n-1} h\right)-\psi_{j}^{n-1}}{h}\right) \psi_{j}^{n-1 *}\right.\right. \\
& \left.\left(\frac{1-\exp \left(-i \kappa\left(a_{j k}^{n}-a_{j k}^{n-1}\right) h\right)}{h}\right)\right\} \\
& +\Re\left\{\psi_{j}^{n-1} \psi_{j}^{n-1 *}\left(\frac{1-\exp \left(-i \kappa\left(a_{j k}^{n}-a_{j k}^{n-1}\right) h\right)-i \kappa\left(a_{j k}^{n}-a_{j k}^{n-1}\right) h}{h^{2}}\right)\right\} \\
& -\Re\left\{i\left(\frac{\psi_{k}^{n} \exp \left(-i \kappa a_{j k}^{n} h\right)-\psi_{j}^{n}}{h}\right) \psi_{j}^{n *} \kappa\left(a_{j k}^{n}-a_{j k}^{n-1}\right)\right\} \\
& \left.-\frac{1}{2}\left(\frac{\left|\left(\psi_{k}^{n}-\psi_{k}^{n-1}\right) \exp \left(-i \kappa a_{j k}^{n} h\right)-\left(\psi_{j}^{n}-\psi_{j}^{n-1}\right)\right|^{2}}{h}\right)\right]
\end{aligned}
$$

where we have used $\Re\left\{i \psi_{j}^{n} \psi_{j}^{n *}\right\}=0$ and $\Re\left\{i \psi_{j}^{n-1} \psi_{j}^{n-1 *}\right\}=0$. It follows that

$$
\begin{aligned}
\mathrm{I}_{1} \leq & \sum_{j k} \frac{h h_{j k}^{\prime}}{\kappa^{2}}\left[\left|\frac{\psi_{k}^{n-1} \exp \left(-i \kappa a_{j k}^{n-1} h\right)-\psi_{j}^{n-1}}{h}\right| \kappa\left|a_{j k}^{n}-a_{j k}^{n-1}\right|+\kappa^{2}\left|a_{j k}^{n}-a_{j k}^{n-1}\right|^{2}\right. \\
& \left.+\left|\frac{\psi_{k}^{n} \exp \left(-i \kappa a_{j k}^{n} h\right)-\psi_{j}^{n}}{h}\right| \kappa\left|a_{j k}^{n}-a_{j k}^{n-1}\right|\right] \\
\leq & \sum_{j k} \frac{h h_{j k}^{\prime}}{\kappa^{2}}\left[\left(\kappa^{2}+\frac{\eta \kappa^{2}}{4 \Delta t}\right)\left|a_{j k}^{n}-a_{j k}^{n-1}\right|^{2}\right. \\
& \left.+\frac{2 \Delta t}{\eta}\left(\frac{\left|\psi_{k}^{n-1} \exp \left(-i \kappa a_{j k}^{n-1} h\right)-\psi_{j}^{n-1}\right|^{2}}{h^{2}}+\frac{\left|\psi_{k}^{n} \exp \left(-i \kappa a_{j k}^{n} h\right)-\psi_{j}^{n}\right|^{2}}{h^{2}}\right)\right]
\end{aligned}
$$

Therefore,

$$
\begin{gathered}
\mathcal{F}^{h}\left(\vec{\psi}^{n}, \vec{A}^{n}\right)-\mathcal{F}^{h}\left(\vec{\psi}^{n-1}, \vec{A}^{n-1}\right)+\frac{1}{\Delta t}\left\{\left\|\vec{\psi}^{n}-\vec{\psi}^{n-1}\right\|_{U, 2}^{2}+\eta\left\|\vec{A}^{n}-\vec{A}^{n-1}\right\|_{W, 2}^{2}\right\} \\
\leq \frac{4 \Delta t}{\eta}\left(\mathcal{F}^{h}\left(\vec{\psi}^{n}, \vec{A}^{n}\right)+\mathcal{F}^{h}\left(\vec{\psi}^{n-1}, \vec{A}^{n-1}\right)\right)+\left(1+\frac{\eta}{4 \Delta t}\right)\left\|\vec{A}^{n}-\vec{A}^{n-1}\right\|_{W, 2}^{2} \\
\quad+\frac{1}{\Delta t}\left\langle\vec{\psi}^{n}-\vec{\psi}^{n-1}, \vec{\zeta}^{n-1}-\vec{\psi}^{n-1}\right\rangle_{U}
\end{gathered}
$$

where $\vec{\zeta}^{n-1}$ is given by

$$
\zeta_{j}^{n-1}=\psi_{j}^{n-1} \exp \left(-i \kappa\left(\Phi_{a j}^{n}+d_{j}^{n}\right) \Delta t\right) .
$$

Since

$$
\begin{gathered}
\frac{1}{\Delta t}\left\|\vec{\zeta}^{n-1}-\vec{\psi}^{n-1}\right\|_{U, 2}^{2} \leq c|\mathbf{J}|^{2} d t+\left\|\vec{d}^{n}\right\|_{U, 2}^{2} \Delta t \\
\leq c|\mathbf{J}|^{2} d t+\mathcal{F}^{h}\left(\vec{\psi}^{n}, \vec{A}^{n}\right) \Delta t
\end{gathered}
$$


we have for $\Delta t$ small enough,

$$
\begin{aligned}
(1- & \left.\frac{4 \Delta t}{\eta}-\frac{\Delta t}{2}\right) \mathcal{F}^{h}\left(\vec{\psi}^{n}, \vec{A}^{n}\right)-\left(1+\frac{4 \Delta t}{\eta}\right) \mathcal{F}^{h}\left(\vec{\psi}^{n-1}, \vec{A}^{n-1}\right) \\
& +\frac{1}{2 \Delta t}\left\{\left\|\vec{\psi}^{n}-\vec{\psi}^{n-1}\right\|_{U, 2}^{2}+\eta\left\|\vec{A}^{n}-\vec{A}^{n-1}\right\|_{W, 2}^{2}\right\} \leq c|\mathbf{J}|^{2} \Delta t .
\end{aligned}
$$

By the discrete Gronwall's inequality, we may conclude that there exists a positive constant $c$, independent of $h$ and $\Delta t$ such that for any $1 \leq n \leq N=T / \Delta t$,

$$
\mathcal{F}^{h}\left(\vec{\psi}^{n}, \vec{A}^{n}\right) \leq c \mathcal{F}^{h}\left(\vec{\psi}^{0}, \vec{A}^{0}\right)+c|\mathbf{J}|^{2} .
$$

Summing the equation (4.9) over $n$ and using (4.10), we get the estimate (4.8).

To complete the estimate, we note that with (3.14c-e) and Lemma 3.2, it is easy to show that for $h$ small,

$$
\mathcal{F}^{h}\left(\vec{\psi}^{0}, \vec{A}^{0}\right) \leq c
$$

for some positive constant $c$, depending only on the initial data and the given parameters $\kappa$ and $H$. So, we have

Corollary 4.3. Given the initial data and the parameters $\kappa, \eta, \mathbf{J}, H$ and $T$, for $2 \leq p<\infty$, there exists a generic positive constant $c$, independent of $h, \Delta t$ and $n$, such that for $h, \Delta t$ small, we have

$$
\begin{aligned}
& \sum_{n=1}^{N} \Delta t\left\{\left\|\frac{\vec{\psi}^{n}-\vec{\psi}^{n-1}}{\Delta t}\right\|_{U, 2}^{2}+\left\|\frac{\vec{A}^{n}-\vec{A}^{n-1}}{\Delta t}\right\|_{W, 2}^{2}\right\} \leq c, \\
& \left\|C \vec{A}^{n}\right\|_{V, 2}+\left\|D \vec{A}^{n}\right\|_{U, 2} \leq c \\
& \left\|\vec{A}^{n}\right\|_{W, p} \leq c \\
& \left\|\nabla \vec{\psi}^{n}\right\|_{W, 2} \leq c \\
& \left\|\vec{\psi}^{n}\right\|_{U, \infty} \leq 1
\end{aligned}
$$

Proof. The first inequality in (4.11) follows from (4.8). Since

$$
\mathcal{F}^{h}\left(\vec{\psi}^{n}, \vec{A}^{n}\right) \leq c,
$$

the second inequality in (4.11) follows from the definition of $\mathcal{F}^{h}$. In turn, the estimate in the $p$ norm for $\vec{A}^{n}$ follows from Lemma 3.2 .

Note that

$$
\begin{gathered}
\frac{1}{\kappa^{2}}\left\|\nabla \vec{\psi}^{n}\right\|_{W, 2}^{2} \leq \sum_{j k} \frac{h h_{j k}^{\prime}}{\kappa^{2}}\left|\frac{\psi_{k}^{n} \exp \left(-i \kappa a_{j k}^{n} h\right)-\psi_{j}^{n}}{h}\right|^{2}+2 \sum_{j k} h h_{j k}^{\prime}\left|\psi_{j}^{n}\right|\left|\psi_{k}^{n} \| a_{j k}^{n}\right|^{2} \\
\leq 2 \mathcal{F}^{h}\left(\vec{\psi}^{n}, \vec{A}^{n}\right)+2\left\|\vec{A}^{n}\right\|_{W, 2}^{2},
\end{gathered}
$$

we get the bound on $\left\|\nabla \vec{\psi}^{n}\right\|_{W, 2}^{2}$.

Finally, the last inequality in (4.11) follows from Lemma 4.1.

Remark. The corresponding version of (4.8) in the zero electric potential gauge (see $\S 3.6$ and replace $\mathcal{F}^{h}$ by $\mathcal{G}^{h}$ ) is much easier to be verified due to (3.16)-(3.17). Nevertheless, it would take more work to establish the estimates similar to (4.11) in that gauge. 
4.3. The comparison of solutions. For a pair of vectors $\left(\vec{f}^{n}, \vec{g}^{n}\right) \in U \times W$, we consider the pair $\left(\vec{\varphi}^{n}, \overrightarrow{\mathfrak{A}}^{n}\right) \in U \times W$ that satisfies

$$
\begin{gathered}
\frac{\varphi_{j}^{n}-\varphi_{j}^{n-1} \exp \left(-i \kappa\left(\Phi_{a j}^{n}+\mathfrak{d}_{j}^{n}\right) \Delta t\right)}{\Delta t}=\left(1-\left|\varphi_{j}^{n}\right|^{2}\right) \varphi_{j}^{n} \\
+\frac{1}{\left|\tau_{j}^{\prime}\right|} \sum_{k \rightarrow j} \frac{h_{j k}^{\prime}}{\kappa^{2}}\left(\frac{\varphi_{k}^{n} \exp \left(-i \kappa \mathfrak{a}_{j k}^{n} h\right)-\varphi_{j}^{n}}{h}\right)+f_{j}^{n}, \\
\eta\left(\frac{\mathfrak{a}_{j k}^{n}-\mathfrak{a}_{j k}^{n-1}}{\Delta t}\right)=\left(\nabla \overrightarrow{\mathfrak{d}}^{n}\right)_{j k}-\left(\nabla^{\perp} \overrightarrow{\mathfrak{c}}^{n}\right)_{j k} \\
+\frac{1}{h \kappa} \Re\left\{i \varphi_{k}^{n *} \varphi_{j}^{n} \exp \left(i \kappa \mathfrak{a}_{j k}^{n} h\right)\right\}+g_{j k}^{n},
\end{gathered}
$$

where $\overrightarrow{\mathfrak{d}}^{n}=D \overrightarrow{\mathfrak{A}}^{n}$ and $\overrightarrow{\mathfrak{c}}^{n}=C \overrightarrow{\mathfrak{A}}^{n}$.

Assume that $\left(\vec{\varphi}^{n}, \overrightarrow{\mathfrak{A}}^{n}\right)$ satisfies the same estimates given in Corollary 4.3 for $\left(\vec{\psi}^{n}, \vec{A}^{n}\right)$ (with the same generic constant $c$ that is independent of $\Delta t$ and $h$ ). Let

$$
\begin{aligned}
& \vec{\alpha}^{n}:=\vec{\psi}^{n}-\vec{\varphi}^{n}, \\
& \vec{e}^{n}:=\vec{A}^{n}-\overrightarrow{\mathfrak{A}}^{n}, \\
& \vec{\gamma}^{n}:=\vec{c}^{n}-\overrightarrow{\mathfrak{c}}^{n}, \\
& \vec{\delta}^{n}:=\vec{d}^{n}-\overrightarrow{\mathfrak{d}}^{n} .
\end{aligned}
$$

We then have

Theorem 4.4. Given the initial data $\left(\vec{\psi}^{0}, \vec{A}^{0}\right)$ and $\left(\vec{\varphi}^{0}, \overrightarrow{\mathfrak{A}}^{0}\right)$ and the parameters $\kappa, \eta, \mathbf{J}, H, T$, there exists a generic constant $c>0$, independent of $h, \Delta t$ and $n$, such that for $h, \Delta t$ small and $1 \leq n \leq N=T / \Delta t$, we have

$$
\begin{gathered}
(1-c \Delta t)\left\|\vec{\alpha}^{n}\right\|_{U, 2}^{2}+(\eta-c \Delta t)\left\|\vec{e}^{n}\right\|_{W, 2}^{2} \leq(1+c \Delta t)\left\|\vec{\alpha}^{n-1}\right\|_{U, 2}^{2} \\
+(\eta+c \Delta t)\left\|\vec{e}^{n-1}\right\|_{W, 2}^{2}+\Delta t\left\|\vec{f}^{n}\right\|_{U, 2}^{2}+\Delta t\left\|\vec{g}^{n}\right\|_{W, 2}^{2} .
\end{gathered}
$$

Proof. Subtracting the equations for the corresponding variables, we get

$$
\begin{aligned}
\frac{\alpha_{j}^{n}-}{\alpha_{j}^{n-1} \exp \left(-i \kappa\left(\Phi_{a j}^{n}+d_{j}^{n}\right) \Delta t\right)} & \Delta t \\
= & \varphi_{j}^{n-1} \exp \left(-i \kappa \Phi_{a j}^{n} \Delta t\right)\left(\frac{\exp \left(-i \kappa \mathfrak{d}_{j}^{n} \Delta t\right)-\exp \left(-i \kappa d_{j}^{n} \Delta t\right)}{\Delta t}\right) \\
& +\left(1-\left|\psi_{j}^{n}\right|^{2}\right) \psi_{j}^{n}-\left(1-\left|\varphi_{j}^{n}\right|^{2}\right) \varphi_{j}^{n} \\
& +\frac{1}{\left|\tau_{j}^{\prime}\right|} \sum_{k \rightarrow j} \frac{h_{j k}^{\prime}}{\kappa^{2}}\left(\frac{\alpha_{k}^{n} \exp \left(-i \kappa a_{j k}^{n} h\right)-\alpha_{j}^{n}}{h}\right) \\
& +\frac{1}{\left|\tau_{j}^{\prime}\right|} \sum_{k \rightarrow j} \frac{h_{j k}^{\prime}}{\kappa^{2}}\left(\frac{\exp \left(-i \kappa e_{j k}^{n} h\right)-1+i \kappa e_{j k}^{n} h}{h}\right) \varphi_{k}^{n} \exp \left(-i \kappa \mathfrak{a}_{j k}^{n} h\right) \\
& -\frac{1}{\left|\tau_{j}^{\prime}\right|} \sum_{k \rightarrow j} \frac{h h_{j k}^{\prime}}{\kappa} i e_{j k}^{n} \frac{\varphi_{k}^{n} \exp \left(-i \kappa \mathfrak{a}_{j k}^{n} h\right)-\varphi_{j}^{n}}{h}-\frac{1}{\left|\tau_{j}^{\prime}\right|} \frac{i}{\kappa} \delta_{j}^{n} \varphi_{j}^{n}-f_{j}^{n},
\end{aligned}
$$


and

$$
\begin{aligned}
& \frac{\eta}{\Delta t}\left(e_{j k}^{n}-e_{j k}^{n-1}\right)=\left(\nabla \vec{\delta}^{n}\right)_{j k}-\left(\nabla^{\perp} \vec{\gamma}^{n}\right)_{j k} \\
& \quad+\frac{1}{h \kappa} \Re\left\{i \alpha_{k}^{n *} \varphi_{j}^{n} \exp \left(i \kappa \mathfrak{a}_{j k}^{n} h\right)+i \varphi_{k}^{n *} \alpha_{j}^{n} \exp \left(i \kappa \mathfrak{a}_{j k}^{n} h\right)\right\} \\
& \quad+\frac{1}{\kappa} \Re\left\{i\left(\alpha_{k}^{n *} \alpha_{j}^{n}-\psi_{k}^{n *} \psi_{j}^{n}\right)\left(\frac{\exp \left(i \kappa \mathfrak{a}_{j k}^{n} h\right)-\exp \left(i \kappa a_{j k}^{n} h\right)}{h}\right)\right\} \\
& \quad+\frac{1}{\kappa} \Re\left\{i \alpha_{k}^{n *}\left(\alpha_{j}^{n} \exp \left(i \kappa a_{j k}^{n} h\right)-\alpha_{k}^{n}\right)\right\}-g_{j k}^{n} .
\end{aligned}
$$

Multiplying (4.14a) by $\left|\tau_{j}^{\prime}\right| \alpha_{j}^{n *}$, taking the real part, and summing over $j$, we get

$$
\begin{aligned}
& \frac{\left\|\vec{\alpha}^{n}\right\|_{U, 2}^{2}-\left\|\vec{\alpha}^{n-1}\right\|_{U, 2}^{2}}{2 \Delta t}+\sum_{j} \frac{\left|\tau_{j}^{\prime}\right|}{2 \Delta t}\left|\alpha_{j}^{n}-\alpha_{j}^{n-1} \exp \left(-i \kappa\left(\Phi_{a j}^{n}+d_{j}^{n}\right) d t\right)\right|^{2} \\
& =\sum_{j}\left|\tau_{j}^{\prime}\right| \Re\left\{\varphi_{j}^{n-1} \alpha_{j}^{n *} \exp \left(-i \kappa \Phi_{a j}^{n} \Delta t\right)\left(\frac{\exp \left(i \kappa \mathfrak{d}_{j}^{n} \Delta t\right)-\exp \left(i \kappa d_{j}^{n} \Delta t\right)}{\Delta t}\right)^{*}\right\} \\
& \quad+\sum_{j}\left|\tau_{j}^{\prime}\right| \Re\left\{\left[\left(1-\left|\psi_{j}^{n}\right|^{2}\right) \psi_{j}^{n}-\left(1-\left|\varphi_{j}^{n}\right|^{2}\right) \varphi_{j}^{n}\right] \alpha_{j}^{n *}\right\} \\
& \quad+\sum_{j} \sum_{k \rightarrow j} \frac{h_{j k}^{\prime}}{\kappa^{2}} \Re\left\{\left(\frac{\alpha_{k}^{n} \exp \left(-i \kappa a_{j k}^{n} h\right)-\alpha_{j}^{n}}{h}\right) \alpha_{j}^{n *}\right\} \\
& \quad+\sum_{j} \sum_{k \rightarrow j} \frac{h_{j k}^{\prime}}{\kappa^{2}} \Re\left\{\left(\frac{\exp \left(-i \kappa e_{j k}^{n} h\right)-1+i \kappa e_{j k}^{n} h}{h}\right) \varphi_{k}^{n} \exp \left(i \kappa \mathfrak{a}_{j k}^{n} h\right) \alpha_{j}^{n *}\right\} \\
& \quad-\sum_{j} \sum_{k \rightarrow j} \frac{h h_{j k}^{\prime}}{\kappa} \Re\left\{i e_{j k}^{n} \frac{\varphi_{k}^{n} \exp \left(-i \kappa \mathfrak{a}_{j k}^{n} h\right)-\varphi_{j}^{n}}{h} \alpha_{j}^{n *}\right\} \\
& \quad-\sum_{j} \frac{\left|\tau_{j}^{\prime}\right|}{\kappa} \delta_{j}^{n} \Re\left\{i \varphi_{j}^{n} \alpha_{j}^{n *}\right\}-\sum_{j}\left|\tau_{j}^{\prime}\right| \Re\left\{f_{j}^{n} \alpha_{j}^{n *}\right\} \\
& \quad=: \mathrm{II}_{1}+\mathrm{II}_{2}+\mathrm{II}_{3}+\mathrm{II}_{4}+\mathrm{II}_{5}+\mathrm{II}_{5}+\mathrm{II}_{6}+\mathrm{II}_{7} .
\end{aligned}
$$

We note that

$$
\begin{aligned}
\mathrm{II}_{1} & :=\sum_{j} \sum_{k \rightarrow j} \frac{h_{j k}^{\prime}}{\kappa^{2}} \Re\left\{\left(\frac{\alpha_{k}^{n} \exp \left(-i \kappa a_{j k}^{n} h\right)-\alpha_{j}^{n}}{h}\right) \alpha_{j}^{n *}\right\} \\
& =-\sum_{j k} \frac{h h_{j k}^{\prime}}{\kappa^{2}}\left|\frac{\alpha_{k}^{n} \exp \left(-i \kappa a_{j k}^{n} h\right)-\alpha_{j}^{n}}{h}\right|^{2} .
\end{aligned}
$$

Moreover,

$$
\begin{aligned}
& -\sum_{j k} \frac{h h_{j k}^{\prime}}{\kappa^{2}}\left|\frac{\alpha_{k}^{n} \exp \left(-i \kappa a_{j k}^{n} h\right)-\alpha_{j}^{n}}{h}\right|^{2} \\
& \quad \leq-\sum_{j k} \frac{h h_{j k}^{\prime}}{\kappa^{2}}\left|\frac{\alpha_{k}^{n}-\alpha_{j}^{n}}{h}\right|^{2}+\left.2 \sum_{j k} h h_{j k}^{\prime}\left|\alpha_{j}^{n}\left\|\alpha_{k}^{n}\right\|\right| a_{j k}^{n}\right|^{2} \\
& \leq-\frac{1}{\kappa^{2}}\left\|\nabla \vec{\alpha}^{n}\right\|_{W, 2}^{2}+8\left\|\vec{\alpha}^{n}\right\|_{U, 4}^{2}\left\|\vec{A}^{n}\right\|_{W, 4}^{2} .
\end{aligned}
$$


Using the uniform estimate on $\left\|\vec{A}^{n}\right\|_{W, 4}$ in Corollary 4.3 and the inequality in Lemma 2.2 with some appropriate $\epsilon$, we get

$$
8\left\|\vec{\alpha}^{n}\right\|_{U, 4}^{2}\left\|\vec{A}^{n}\right\|_{W, 4}^{2} \leq \frac{1}{2 \kappa^{2}}\left\|\nabla \vec{\alpha}^{n}\right\|_{W, 2}^{2}+c\left\|\vec{\alpha}^{n}\right\|_{U, 2}^{2}
$$

for some generic constant $c$. Thus,

$$
\mathrm{II}_{1} \leq-\frac{1}{2} \sum_{j k} \frac{h h_{j k}^{\prime}}{\kappa^{2}}\left|\frac{\alpha_{k}^{n} \exp \left(-i \kappa a_{j k}^{n} h\right)-\alpha_{j}^{n}}{h}\right|^{2}-\frac{1}{2 \kappa^{2}}\left\|\nabla \vec{\alpha}^{n}\right\|_{W, 2}^{2}+c\left\|\vec{\alpha}^{n}\right\|_{U, 2}^{2} .
$$

Concerning other terms in the equation (4.15), we have

$$
\begin{aligned}
& \mathrm{II}_{2}:=\frac{1}{\Delta t} \sum_{j}\left|\tau_{j}^{\prime}\right| \mid \Re\left\{\varphi_{j}^{n-1} \alpha_{j}^{n *} \exp \left(-i \kappa \Phi_{a j}^{n} \Delta t\right)\right. \\
&\left.\left(\exp \left(-i \kappa \mathfrak{d}_{j}^{n} \Delta t\right)-\exp \left(-i \kappa d_{j}^{n} \Delta t\right)\right)\right\} \mid \\
& \leq \sum_{j}\left|\tau_{j}^{\prime}\left\|\alpha_{j}^{n}|| \delta_{j}^{n} \mid \leq \frac{1}{4}\right\| \vec{\delta}^{n}\left\|_{U, 2}^{2}+\right\| \vec{\alpha}^{n} \|_{U, 2}^{2} .\right.
\end{aligned}
$$

The estimate $\left|\varphi_{j}^{n-1}\right| \leq 1$ is used in the above. Next, using monotonicity, we have

$$
\mathrm{II}_{3}:=\sum_{j}\left|\tau_{j}^{\prime}\right| \Re\left\{\left[\left(1-\left|\psi_{j}^{n}\right|^{2}\right) \psi_{j}^{n}-\left(1-\left|\varphi_{j}^{n}\right|^{2}\right) \varphi_{j}^{n}\right] \alpha_{j}^{n *}\right\} \leq 0 .
$$

Noting that $\left|\alpha_{j}^{n}\right| \leq 2$ and $\left|\varphi_{k}^{n}\right| \leq 1$ we have

$$
\begin{aligned}
\mathrm{II}_{4} & :=\sum_{j} \sum_{k \rightarrow j} \frac{h h_{j k}^{\prime}}{\kappa^{2}}\left|\Re\left\{\left(\frac{\exp \left(-i \kappa e_{j k}^{n} h\right)-1+i \kappa e_{j k}^{n} h}{h^{2}}\right) \varphi_{k}^{n} \exp \left(-i \kappa \mathfrak{a}_{j k}^{n} h\right) \alpha_{j}^{n *}\right\}\right| \\
& \leq \sum_{j} \sum_{k \rightarrow j} h h_{j k}^{\prime}\left|e_{j k}^{n}\right|^{2}\left|\alpha_{j}^{n}\right| \leq 4\left\|\vec{e}^{n}\right\|_{W, 2}^{2} .
\end{aligned}
$$

Also,

$$
\begin{aligned}
\mathrm{II}_{5} & :=\sum_{j} \sum_{k \rightarrow j} \frac{h h_{j k}^{\prime}}{\kappa}\left|\Re\left\{i e_{j k}^{n} \frac{\varphi_{k}^{n} \exp \left(-i \kappa \mathfrak{a}_{j k}^{n} h\right)-\varphi_{j}^{n}}{h} \alpha_{j}^{n *}\right\}\right| \\
& \leq \sum_{j} \sum_{k \rightarrow j} \frac{h h_{j k}^{\prime}}{\kappa}\left|e_{j k}^{n} \| \alpha_{j}^{n}\right|\left|\frac{\varphi_{k}^{n} \exp \left(-i \kappa \mathfrak{a}_{j k}^{n} h\right)-\varphi_{j}^{n}}{h}\right| \\
& \leq 4 \mathcal{F}^{h}\left(\vec{\varphi}^{n}, \overrightarrow{\mathfrak{A}}^{n}\right)^{1 / 2}\left\|\vec{\alpha}^{n}\right\|_{U, 4}\left\|\vec{e}^{n}\right\|_{W, 4} .
\end{aligned}
$$

Using the uniform bound on $\mathcal{F}^{h}\left(\vec{\varphi}^{n}, \overrightarrow{\mathfrak{A}}^{n}\right)$ and the inequalities in Lemmas 2.2 and 3.2 , we get for some generic constant $c>0$,

$$
\begin{aligned}
\mathrm{II}_{5} & \leq 4 \mathcal{F}^{h}\left(\vec{\varphi}^{n}, \overrightarrow{\mathfrak{A}}^{n}\right)^{1 / 2}\left\|\vec{\alpha}^{n}\right\|_{U, 4}\left\|\vec{e}^{n}\right\|_{W, 4} \\
& \leq \frac{1}{6 \kappa^{2}}\left\|\nabla \vec{\alpha}^{n}\right\|_{W, 2}^{2}+\frac{1}{4}\left\|\delta^{n}\right\|_{U, 2}^{2}+\frac{1}{4}\left\|\gamma^{n}\right\|_{V, 2}^{2}+c\left\|\vec{\alpha}^{n}\right\|_{U, 2}^{2} .
\end{aligned}
$$

In addition, we have

$$
\begin{aligned}
\mathrm{II}_{6} & :=\sum_{j} \frac{\left|\tau_{j}^{\prime}\right|}{\kappa}\left|\Re\left\{i \delta_{j}^{n} \varphi_{j}^{n} \alpha_{j}^{n *}\right\}\right| \leq \frac{1}{\kappa} \sum_{j}\left|\tau_{j}^{\prime}\left\|\delta_{j}^{n}\right\|\right| \alpha_{j}^{n} \mid \\
& \leq \frac{1}{\kappa^{2}}\left\|\vec{\alpha}^{n}\right\|_{U, 2}^{2}+\frac{1}{4}\left\|\vec{\delta}^{n}\right\|_{U, 2}^{2}
\end{aligned}
$$


and

$$
\mathrm{II}_{7}:=\sum_{j}\left|\tau_{j}^{\prime}\right|\left|\Re\left\{f_{j}^{n} \alpha_{j}^{n *}\right\}\right| \leq \frac{1}{2}\left\|\vec{\alpha}^{n}\right\|_{U, 2}^{2}+\frac{1}{2}\left\|\vec{f}^{n}\right\|_{U, 2}^{2} .
$$

By the above estimates, (4.15) implies

$$
\begin{aligned}
& \frac{1}{2 \Delta t}\left(\left\|\vec{\alpha}^{n}\right\|_{U, 2}^{2}-\left\|\vec{\alpha}^{n-1}\right\|_{U, 2}^{2}\right)+\frac{1}{3 \kappa^{2}}\left\|\nabla \vec{\alpha}^{n}\right\|_{W, 2}^{2} \\
& \quad+\frac{1}{2} \sum_{j k} \frac{h h_{j k}^{\prime}}{\kappa^{2}}\left|\frac{\alpha_{k}^{n} \exp \left(-i \kappa a_{j k}^{n} h\right)-\alpha_{j}^{n}}{h}\right|^{2} \\
& \leq c\left\|\vec{\alpha}^{n}\right\|_{U, 2}^{2}+4\left\|\vec{e}^{n}\right\|_{W, 2}^{2}+\frac{1}{2}\left\|\vec{\delta}^{n}\right\|_{U, 2}^{2}+\frac{1}{4}\left\|\vec{\gamma}^{n}\right\|_{V, 2}^{2}+\frac{1}{2}\left\|\vec{f}^{n}\right\|_{U, 2}^{2} .
\end{aligned}
$$

Meanwhile, multiplying $e_{j k}^{n} h h_{j k}^{\prime}$ to the equation (4.14b) and summing over all edges $s_{j k}$ and using summation by parts (see Lemma 3.1), we get

$$
\begin{aligned}
& \frac{\eta}{2 \Delta t}\left(\left\|\vec{e}^{n}\right\|_{W, 2}^{2}-\left\|\vec{e}^{n-1}\right\|_{W, 2}^{2}+\left\|\vec{e}^{n}-\vec{e}^{n-1}\right\|_{W, 2}^{2}\right)+\left\|\vec{\delta}^{n}\right\|_{U, 2}^{2}+\left\|\vec{\gamma}^{n}\right\|_{V, 2}^{2} \\
& \quad=\sum_{j k} \frac{h_{j k}^{\prime}}{\kappa} \Re\left\{i \alpha_{k}^{n *} \varphi_{j}^{n} \exp \left(i \kappa \mathfrak{a}_{j k}^{n} h\right) e_{j k}^{n}+i \varphi_{k}^{n *} \alpha_{j}^{n} \exp \left(i \kappa a_{j k}^{n} h\right) e_{j k}^{n}\right\} \\
& \quad+\sum_{j k} \frac{h h_{j k}^{\prime}}{\kappa} \Re\left\{i\left(\psi_{k}^{n *} \psi_{j}^{n}-\alpha_{k}^{n *} \alpha_{j}^{n}\right)\left(\frac{\exp \left(i \kappa a_{j k}^{n} h\right)-\exp \left(i \kappa \mathfrak{a}_{j k}^{n} h\right)}{h}\right) e_{j k}^{n}\right\} \\
& \quad+\sum_{j k} \frac{h h_{j k}^{\prime}}{\kappa} \Re\left\{i\left(\alpha_{k}^{n *}\left(\frac{\alpha_{j}^{n} \exp \left(i \kappa a_{j k}^{n} h\right)-\alpha_{k}^{n}}{h}\right) e_{j k}^{n}\right\}-\sum_{j k} h h_{j k}^{\prime} g_{j}^{n} e_{j k}^{n}\right. \\
& \quad=: \mathrm{III}_{1}+\mathrm{III}_{2}+\mathrm{III}_{3}+\mathrm{III}_{4} .
\end{aligned}
$$

Note that

$$
\begin{aligned}
\mathrm{III}_{1} & :=\sum_{j k} \frac{h_{j k}^{\prime}}{\kappa} \Re\left\{i \alpha_{k}^{n *} \varphi_{j}^{n} \exp \left(i \kappa \mathfrak{a}_{j k}^{n} h\right) e_{j k}^{n}+i \varphi_{k}^{n *} \alpha_{j}^{n} \exp \left(i \kappa a_{j k}^{n} h\right) e_{j k}^{n}\right\} \\
& =-\sum_{j} \sum_{k \rightarrow j} \frac{h_{j k}^{\prime}}{\kappa} \Re\left\{i e_{j k}^{n} \varphi_{k}^{n} \exp \left(i \kappa \mathfrak{a}_{j k}^{n} h\right) \alpha_{j}^{n *}\right\} \\
& \leq \frac{1}{6 \kappa^{2}}\left\|\nabla \vec{\alpha}^{n}\right\|_{W, 2}^{2}+\frac{1}{4}\left\|\delta^{n}\right\|_{U, 2}^{2}+\frac{1}{4}\left\|\gamma^{n}\right\|_{V, 2}^{2}+c\left\|\vec{\alpha}^{n}\right\|_{U, 2}^{2}
\end{aligned}
$$

by the derivation given earlier. Using the pointwise estimates $\left|\psi_{j}^{n}\right| \leq 1,\left|\psi_{k}^{n}\right| \leq 1$, $\left|\alpha_{j}^{n}\right| \leq 2$ and $\left|\alpha_{k}^{n}\right| \leq 2$, we get

$$
\begin{aligned}
\mathrm{III}_{2}:= & \sum_{j k} \frac{h h_{j k}^{\prime}}{\kappa}\left|\Re\left\{i\left(\psi_{k}^{n *} \psi_{j}^{n}-\alpha_{k}^{n *} \alpha_{j}^{n}\right)\left(\frac{\exp \left(i \kappa a_{j k}^{n} h\right)-\exp \left(i \kappa \mathfrak{a}_{j k}^{n} h\right)}{h}\right) e_{j k}^{n}\right\}\right| \\
& \leq 5\left\|\vec{e}^{n}\right\|_{W, 2}^{2} .
\end{aligned}
$$

Also,

$$
\begin{aligned}
\mathrm{III}_{3} & :=\sum_{j k} \frac{h h_{j k}^{\prime}}{\kappa}\left|\Re\left\{i\left(\alpha_{k}^{n *}\right)\left(\frac{\alpha_{j}^{n} \exp \left(i \kappa a_{j k}^{n} h\right)-\alpha_{k}^{n}}{h}\right) e_{j k}^{n}\right\}\right| \\
& \leq 2\left\|\vec{e}^{n}\right\|_{W, 2}^{2}+\sum_{j k} \frac{h h_{j k}^{\prime}}{2 \kappa^{2}}\left|\frac{\alpha_{k}^{n} \exp \left(-i \kappa a_{j k}^{n} h\right)-\alpha_{j}^{n}}{h}\right|^{2} .
\end{aligned}
$$


Clearly,

$$
\mathrm{III}_{4}:=\sum_{j k} h h_{j k}^{\prime}\left|g_{j k}^{n} e_{j k}^{n}\right| \leq \frac{1}{2}\left\|\vec{e}^{n}\right\|_{W, 2}^{2}+\frac{1}{2}\left\|\vec{g}^{n}\right\|_{W, 2}^{2} .
$$

Thus, (4.17) implies

$$
\begin{aligned}
& \frac{\eta}{2 \Delta t}\left(\left\|\vec{e}^{n}\right\|_{W, 2}^{2}-\left\|\vec{e}^{n-1}\right\|_{W, 2}^{2}+\left\|\vec{e}^{n}-\vec{e}^{n-1}\right\|_{W, 2}^{2}\right)+\frac{3}{4}\left\|\vec{\delta}^{n}\right\|_{U, 2}^{2}+\frac{3}{4}\left\|\vec{\gamma}^{n}\right\|_{V, 2}^{2} \\
& \leq 6\left\|\vec{e}^{n}\right\|_{W, 2}^{2}+\frac{1}{2}\left\|\vec{g}^{n}\right\|_{W, 2}^{2}+\frac{1}{6 \kappa^{2}}\left\|\nabla \vec{\alpha}^{n}\right\|_{W, 2}^{2}+c\left\|\vec{\alpha}^{n}\right\|_{U, 2}^{2} \\
& \quad+\frac{1}{2} \sum_{j k} \frac{h h_{j k}^{\prime}}{\kappa^{2}}\left|\frac{\alpha_{k}^{n} \exp \left(-i \kappa a_{j k}^{n} h\right)-\alpha_{j}^{n}}{h}\right|^{2} .
\end{aligned}
$$

Finally, combining (4.16) with (4.18), we have

$$
\begin{aligned}
& \frac{1}{\Delta t}\left(\left\|\vec{\alpha}^{n}\right\|_{U, 2}^{2}-\left\|\vec{\alpha}^{n-1}\right\|_{U, 2}^{2}\right)+\frac{\eta}{\Delta t}\left(\left\|\vec{e}^{n}\right\|_{W, 2}^{2}-\left\|\vec{e}^{n-1}\right\|_{W, 2}^{2}\right) \\
& \quad \leq c\left\|\vec{\alpha}^{n}\right\|_{U, 2}^{2}+c\left\|\vec{e}^{n}\right\|_{W, 2}^{2}+\left\|\vec{f}^{n}\right\|_{U, 2}^{2}+\left\|\vec{g}^{n}\right\|_{W, 2}^{2}
\end{aligned}
$$

for some constant $c$, independent of $\Delta t$ and $h$. Hence,

$$
\begin{aligned}
& (1-c \Delta t)\left\|\vec{\alpha}^{n}\right\|_{U, 2}^{2}+(\eta-c \Delta t)\left\|\vec{e}^{n}\right\|_{W, 2}^{2} \\
& \quad \leq(1+c \Delta t)\left\|\vec{\alpha}^{n-1}\right\|_{U, 2}^{2}+(\eta+c \Delta t)\left\|\vec{e}^{n-1}\right\|_{W, 2}^{2}+\Delta t\left\|\vec{f}^{n}\right\|_{U, 2}^{2}+\Delta t\left\|\vec{g}^{n}\right\|_{W, 2}^{2} .
\end{aligned}
$$

We see that (4.13) is valid for small $h$ and $\Delta t$.

It follows from the discrete Gronwall's inequality that

Corollary 4.5. Let the initial data $\left(\vec{\psi}^{0}, \vec{A}^{0}\right)$ and $\left(\vec{\varphi}^{0}, \overrightarrow{\mathfrak{A}}^{0}\right)$ and the parameters $\kappa, \eta$, $\mathbf{J}, H, T$ be given. For $h, \Delta t$ small, there exists some constant $c>0$, independent of $h, \Delta t$ and $n$, such that for any $1 \leq n \leq N=T / \Delta t$, we have

$$
\left\|\vec{\alpha}^{n}\right\|_{U, 2}^{2}+\left\|\vec{e}^{n}\right\|_{W, 2}^{2} \leq c\left(\left\|\vec{\alpha}^{0}\right\|_{U, 2}^{2}+\left\|\vec{e}^{0}\right\|_{W, 2}^{2}\right)+c \sum_{l=1}^{n} \Delta t\left(\left\|\vec{f}^{n}\right\|_{U, 2}^{2}+\left\|\vec{g}^{n}\right\|_{W, 2}^{2}\right) .
$$

Remark. One may view the estimate (4.20) as a stability property for the fullydiscrete schemes in the Lorentz gauge. The result is independent of the regularity estimates on the solution of the continuous system (2.1a-f).

4.4. Uniqueness of the discrete solution. The comparison estimates obtained previously have a number of consequences. First of all, we have the uniqueness of the fully discrete solution in the Lorentz gauge.

Theorem 4.6. For small $\Delta t$ and $h$, the scheme (4.4a-b) has a unique solution.

Proof. Let $\vec{f}^{n}=\overrightarrow{0}, \vec{g}^{n}=\overrightarrow{0}$. Assume that for some $n \geq 1, \vec{\varphi}^{n-1}=\vec{\psi}^{n-1}$ and $\overrightarrow{\mathfrak{A}}^{n-1}=\vec{A}^{n-1}$. If both $\left(\vec{\varphi}^{n}, \overrightarrow{\mathfrak{A}}^{n}\right)$ and $\left(\vec{\psi}^{n}, \vec{A}^{n}\right)$ are solutions of the fully-discrete scheme, then we have from Theorem 4.4 that

$$
\left\|\vec{\psi}^{n}-\vec{\varphi}^{n}\right\|_{U, 2}^{2}+\left\|\vec{A}^{n}-\overrightarrow{\mathfrak{A}}^{n}\right\|_{W, 2}^{2} \leq 0 .
$$

Thus, $\vec{\psi}^{n}=\vec{\varphi}^{n}$ and $\vec{A}^{n}=\overrightarrow{\mathfrak{A}}^{n}$. Thus, the solution is unique.

The above result indicates that the Lorentz gauge is indeed a valid gauge choice in the sense that with this gauge choice, there exists one and only one solution to the discrete approximation scheme. 
4.5. Error estimate. In the following, we assume that the exact solution $(\psi, \mathbf{A})$ of $(2.1 \mathrm{a}-\mathrm{f})$ in the Lorentz gauge is sufficiently regular. At $t_{n}=n \Delta t$, let the components of $\vec{\varphi}^{n}$ be defined by

$$
\varphi_{j}^{n}:=\psi\left(\mathbf{x}_{j}, t_{n}\right)
$$

and the components of $\overrightarrow{\mathfrak{A}}^{n}$ be defined by

$$
\mathfrak{a}_{j k}^{n}:=\int_{0}^{1} \mathbf{A}\left((1-s) \mathbf{x}_{j}+s \mathbf{x}_{k}, t_{n}\right) \cdot \mathbf{t}_{j k} d s .
$$

Using a priori estimates for the time dependent GL equations (2.1a-f) similar to the ones in [5], we can easily verify that, by properly choosing (or redefining) the generic constant $c,\left(\vec{\varphi}^{n}, \overrightarrow{\mathfrak{A}}^{n}\right)$ satisfies the same estimates given in Corollary 4.3 as its gauge invariant approximation. Let $\overrightarrow{\mathfrak{d}}^{n}=D \overrightarrow{\mathfrak{A}}^{n}$ and $\overrightarrow{\mathfrak{c}}^{n}=C \overrightarrow{\mathfrak{A}}^{n}$. Then, direct calculation shows that

$$
\begin{gathered}
\frac{\varphi_{j}^{n}-\varphi_{j}^{n-1} \exp \left(-i \kappa\left(\Phi_{a j}^{n}+\mathfrak{d}_{j}^{n}\right) \Delta t\right)}{\Delta t}=\left(1-\left|\varphi_{j}^{n}\right|^{2}\right) \varphi_{j}^{n} \\
+\frac{1}{\left|\tau_{j}^{\prime}\right|} \sum_{k \rightarrow j} \frac{h_{j k}^{\prime}}{\kappa^{2}}\left(\frac{\varphi_{k}^{n} \exp \left(-i \kappa \mathfrak{a}_{j k}^{n} h\right)-\varphi_{j}^{n}}{h}\right)+f_{j}^{n}, \\
\eta\left(\frac{\mathfrak{a}_{j k}^{n}-\mathfrak{a}_{j k}^{n-1}}{\Delta t}\right)=\left(\nabla \overrightarrow{\mathfrak{d}}^{n}\right)_{j k}-\left(\nabla^{\perp}\left(\overrightarrow{\mathfrak{c}}^{n}-\vec{H}\right)\right)_{j k} \\
+\frac{1}{h \kappa} \Re\left\{i \varphi_{k}^{n *} \varphi_{j}^{n} \exp \left(i \kappa \mathfrak{a}_{j k}^{n} h\right)\right\}+g_{j k}^{n},
\end{gathered}
$$

for some $\vec{f}^{n}$ and $\vec{g}^{n}$ which satisfy

$$
\sum_{l=1}^{n} \Delta t\left(\left\|\vec{f}^{n}\right\|_{U, 2}^{2}+\left\|\vec{g}^{n}\right\|_{W, 2}^{2}\right) \leq c\left(\Delta t^{2}+h^{4}\right), \text { for } n=1,2, \ldots, N
$$

where $c$ is a positive constant depending only on the appropriate norms of the exact solution $(\psi, \mathbf{A})$. One may consult [5], [13], [24] for the regularity estimates for the weak and strong solutions of the time dependent GL equations in various gauges.

Using a similar error estimate as those given in [22], [23], we have

$$
\left\|\vec{A}^{0}-\overrightarrow{\mathfrak{A}}^{0}\right\|_{W, 2} \leq c h^{2}\left\|\mathbf{A}_{0}\right\|_{2, \Omega},
$$

for the initial data $\mathbf{A}_{0} \in \mathbf{H}^{2}(\Omega)$. By Corollary 4.5, we get from (4.23) and (4.24) that

Theorem 4.7. There exists a positive constant $c$ which depends on the norms of the exact solution $(\psi, \mathbf{A})$ and the given parameters $\kappa, \eta, \mathbf{J}, H$ and the final time $T$, but is independent of $h$ and $\Delta t$, such that if $(\psi, \mathbf{A})$ is sufficiently smooth and $\Delta t$ and $h$ are sufficiently small, then for any $1 \leq n \leq N$, we have

$$
\left\|\vec{\psi}^{n}-\vec{\varphi}^{n}\right\|_{U, 2}+\left\|\vec{A}^{n}-\overrightarrow{\mathfrak{A}}^{n}\right\|_{W, 2} \leq c\left(\Delta t+h^{2}\right)
$$

where $\left(\vec{\varphi}^{n}, \overrightarrow{\mathfrak{A}}^{n}\right)$ is defined from $(\psi, \mathbf{A})$ by $(4.21 a-b)$.

When variable time step is used, similar estimates may be derived. Error estimates may also be obtained for approximations in other gauges as well as the semi-discrete gauge invariant approximation. 


\section{Conclusion}

The discrete gauge invariant difference approximation is a popular method used by physicists and mathematicians to study the vortex phenomena in superconductors. The above discussion provides a rigorous mathematical theory for this method. The techniques presented here may be applied to analyze other types of fully-discrete schemes, such as the explicit time-stepping scheme used in [17] and other more efficient implementation of the nonlinear coupling. By generalizing the ideas given here and [12], one may also develop and analyze a covolume approximation for the time dependent GL equations on general triangular grids. The numerical implementation of the discrete gauge invariant approximation has already been used in simulations of three dimensional problems (e.g. [3], [16]). One may also try to generalize the theory to such cases. In addition, the pointwise estimate on the order parameter $\psi$ proved here has not been shown for the finite element approximation of the time dependent Ginzburg-Landau equations. The above discussion may provide some hints on how to modify the standard finite element methods by using proper coupling of the nonlinear terms and the quadrature formula to insure the validity of the pointwise bound on the finite element approx-

imation of the order parameter $\psi$. Further studies of the above issues are under way.

\section{ACKNOWLEDGMENT}

The author would like to thank an anonymous referee for many helpful suggestions.

\section{REFERENCES}

1. S. L. Adler, and T. Piran, Relaxation methods for gauge field equilibrium equations, Rev. Mod. Phys. 56 (1984), pp. 1-40. MR 85c:81022

2. Z. Chen, and K.-H. Hoffmann, Numerical studies of a nonstationary Ginzburg-Landau model for superconductivity, Adv. Math. Sci. Appl. 5(1995), 363-389. MR 97e:82051

3. G. Crabtree, G. Leaf, H. Kaper, V. Vinokur, A. Koshelev, D. Braun, D. Levine, W. Kkwok, and J. Fendrich, Time-dependent Ginzburg-Landau simulations of vortex guidance by twin boundaries, Physica C., 263(1996), pp. 401-408.

4. M. Doria, J. Gubernatis, and D. Rainer, Solving the Ginzburg-Landau equations by simulated annealing, Phys. Rev. B, 41 (1990), pp. 6335-6340.

5. Q. Du, Global existence and uniqueness of solutions for the time dependent Ginzburg-Landau model of superconductivity, Applicable Analysis, 53 (1994), 1-2, pp. 1-17. MR 97a:82085

6. Q. Du, Finite element methods for the time dependent Ginzburg-Landau model of superconductivity, Comp. Math. Appl., 27 (1994), pp. 119-133. MR 95g:65131

7. Q. Du, M. Gunzburger, and J. Peterson, Analysis and approximation of Ginzburg-Landau models for superconductivity, SIAM Review, 34(1992), pp. 54-81. MR 93g:82109

8. Q. Du, M. Gunzburger, and J. Peterson, Finite element approximation of a periodic GinzburgLandau model for type-II superconductors, Numer. Math., 64(1993), pp. 85-114. MR 93j:65178

9. Q. Du, M. Gunzburger, and J. Peterson, Solving the Ginzburg-Landau equations by finite element methods, Phy. Rev. B., 46(1992), pp. 9027-9034.

10. Q. Du, M. Gunzburger, and J. Peterson, Computational simulation of type-II superconductivity including pinning phenomena, Phy. Rev. B., 51(1995), pp. 16194-16203.

11. F. Lin and Q. Du, Ginzburg-Landau vortices, dynamics, pinning and hysteresis, SIAM Math. Anal. 28 (1997), pp. 1265-1293. CMP 98:02

12. Q. Du, R. Nicolaides, and $\mathrm{X}$. Wu, Analysis and convergence of a covolume approximation of the Ginzburg-Landau model of superconductivity, to appear in SIAM Numer. Anal., (1997). 
13. J. Fleckinger-Pelle, H. Kaper, and P. Takac, Dynamics of the Ginzburg-Landau equations of superconductivity, preprint, MCS P565-0296, Argonne National Lab..

14. H. Frahm, S. Ullah and A. Dorsey, Flux dynamics and the growth of the superconducting phase, Phys. Rev. Letters, 66 (1991), pp. 3067-3072.

15. L. Freitag, M. Jones and P. Plassmann, New techniques for parallel simulation of high temperature superconductors, MCS preprint, Argonne National Lab., (1994).

16. J. Garner, M. Spanbauer, R. Benedek, K. Strandburg, S. Wright and P. Plassman, Critical fields of Josephson-coupled superconducting multilayers, preprint.

17. W. Gropp, H. Kaper, G. Leaf, D. Levine, M. Plumbo, and V. Vinokur, Numerical simulation of vortex dynamics in type-II superconductors, J. Comp. Phys. 123(1996), pp. 254-266. CMP 96:07

18. H. Kaper, and M. Kwong, Vortex configurations in type-II superconducting films, J. Com. Phys. 119(1995), pp. 120-131.

19. M. K. Wong, Sweeping algorithms for inverting the discrete Ginzburg-Landau operators, Applied Math. Comp., 53(1993), pp.12d90-150.

20. S. Lin and Y. Yang, Computation of superconductivity in thin films, J. Comp. Phys., 89(1990), pp. 257-275. MR 91g:82059

21. F. Liu, M. Mondello and N. Goldenfeld, Kinetics of the superconducting transition, Phys. Rev. Lett., 66 (1991), pp. 3071-3074.

22. R. Nicolaides, Direct discretization of planar div-curl problems, SIAM Numer. Anal., 29 (1992), pp. 32-56. MR 93b:65176

23. R. Nicolaides, and $\mathrm{X}$. Wu, Analysis and convergence of the MAC scheme 2. Navier-Stokes equations, Math. Comp., 65(1996), pp. 29-44. MR 96d:65148

24. Q. Tang and S. Wang, Time-dependent Ginzburg-Landau equations of superconductivity, Physica D., 88(1995), pp. 139-166. MR 96i:82156

25. M. Tinkham, Introduction to Superconductivity, 2nd edition, McGraw-Hill, New York, 1994.

Department of Mathematics, Hong Kong University of Science and Technology, Clear Water Bay, Kowloon, Hong Kong and Department of Mathematics, Iowa State University, Ames, IOWA 50011

E-mail address: madu@uxmail.ust.hk 